\title{
Systolic volume of homology classes
}

\author{
IVAN BABENKO \\ FLORENT BALACHEFF
}

\begin{abstract}
Given an integer homology class of a finitely presentable group, the systolic volume quantifies how tight a geometric realization of this class could be. In this paper, we study various aspects of this numerical invariant showing that it is a complex and powerful tool for investigating topological properties of homology classes of finitely presentable groups.
\end{abstract}

53C23; 20J06, 20F99

\section{Introduction}

Let $G$ be a finitely presentable group and fix a nontrivial homology class $\boldsymbol{a} \in H_{m}(G, \mathbb{Z})$ of dimension $m \geq 1$. In order to study topological and algebraic properties of the class $\boldsymbol{a}$, we consider the various ways this class can be realized by a pseudomanifold endowed with a polyhedral metric. For such realizations, the two main geometrical quantities are the volume of the pseudomanifold and the lengths of loops representing nontrivial elements of $G$. The systolic volume turns out to be the simplest way to compare these geometric quantities in order to form a topological invariant and is defined as follows.

A geometric cycle $(X, f)$ representing a class $\boldsymbol{a}$ is a pair $(X, f)$ consisting of an orientable pseudomanifold $X$ of dimension $m$ and a continuous map $f: X \rightarrow K(G, 1)$ such that $f_{*}[X]=\boldsymbol{a}$, where $[X]$ denotes the fundamental class of $X$ and $K(G, 1)$ the Eilenberg-Mac Lane space. ${ }^{1}$ The representation is said to be normal if in addition the induced map $f_{\sharp}: \pi_{1}(X) \rightarrow G$ is an epimorphism. Given a geometric cycle $(X, f)$ and a polyhedral metric $g$ on $X$ (see Babenko [3] for a definition), the relative homotopic systole $\operatorname{sys}_{f}(X, g)$ is defined as the least length of a loop $\gamma$ in $X$ such that $f \circ \gamma$ is not contractible. The systolic volume of $(X, f)$ is then the value

$$
\mathfrak{S}_{f}(X):=\inf _{g} \frac{\operatorname{vol}(X, g)}{\operatorname{sys}_{f}(X, g)^{m}}
$$

${ }^{1}$ This definition slightly differs from the original one by Gromov in [17], where in addition a geometric cycle is provided with a polyhedral metric on $X$. 
where the infimum is taken over all polyhedral metrics $g$ on $X$ and $\operatorname{vol}(X, g)$ denotes the $m$-dimensional volume of $X$. When $f: X \rightarrow K\left(\pi_{1}(X), 1\right)$ is the classifying map (induced by an isomorphism between fundamental groups), we simply denote by $\mathfrak{S}(X)$ the systolic volume of the pair $(X, f)$. From [17, Section 6], we have that for any dimension $m \geq 1$,

$$
\sigma_{m}:=\inf _{(X, f)} \mathfrak{S}_{f}(X)>0,
$$

the infimum being taken over all geometric cycles $(X, f)$ representing a nontrivial homology class of dimension $m$. The following notion was introduced by Gromov (see [17, Section 6]):

Definition The systolic volume of the pair $(G, \boldsymbol{a})$ is defined as the number

$$
\mathfrak{S}(G, \boldsymbol{a}):=\inf _{(X, f)} \mathfrak{S}_{f}(X),
$$

where the infimum is taken over all geometric cycles $(X, f)$ representing the class $\boldsymbol{a}$.

Any integer class admits a representation by a geometric cycle; see Theorem I below. The systolic volume of $(G, \boldsymbol{a})$ is thus well-defined and satisfies $\mathfrak{S}(G, \boldsymbol{a}) \geq \sigma_{m}$. But it is not clear whether the infimum value $\mathfrak{S}(G, \boldsymbol{a})$ is actually a minimum and what the structure of a minimal geometric cycle would be. When the class $\boldsymbol{a}$ is representable by a manifold, the systolic volume coincides with the systolic volume of any normal representation of $\boldsymbol{a}$ by a manifold; see Babenko [3; 4] and Brunnbauer [10]. A manifold is an example of admissible pseudomanifold, that is a special type of pseudomanifolds for which any element of the fundamental group can be represented by a curve not going through the singular locus of $X$. In this article, we first prove the following result; see Section 2.

Theorem I Let $G$ be a finitely presentable group. Any homology class $\boldsymbol{a} \in H_{m}(G, \mathbb{Z})$ admits a normal representation by an admissible geometric cycle. Furthermore, any such representation by an admissible geometric cycle $(X, f)$ is minimal in the sense that

$$
\mathfrak{S}_{f}(X)=\mathfrak{S}(G, \boldsymbol{a}) .
$$

Here an admissible geometric cycle $(X, f)$ stands for a geometric cycle whose pseudomanifold $X$ is admissible. Thus the infimum in the definition of systolic volume of a homology class is a minimum. Furthermore the systolic volume of an admissible orientable pseudomanifold $X$ depends only on the image of its fundamental class $f_{*}[X] \in H_{m}\left(\pi_{1}(X), \mathbb{Z}\right)$. In Section 6 we exhibit an example showing that the condition of normalization (that is, $f_{\sharp}$ is an epimorphism between fundamental groups) cannot be 
relaxed in our theorem. Finally observe that working with the class of pseudomanifolds is necessary and not merely a formal generalization: there exist classes that are not representable by manifolds; see Thom [27].

In order to understand the systolic volume invariant, we study its distribution along the real line. Here two phenomena appear. First, the systolic volume function does not avoid arbitrarily large intervals.

Proposition I Let $m \geq 3$. For any interval $I \subset \mathbb{R}^{+}$of length at least $\sigma_{m}$, there exists a pair $(G, \boldsymbol{a})$ consisting of a finitely presentable group and a homology class of dimension $m$ such that $\mathfrak{S}(G, \boldsymbol{a}) \in I$.

Secondly, there is no finiteness result for systolic volume in dimension $m \geq 3$. In order to avoid irrelevant nonfiniteness results, we introduce the following definition. A class $\boldsymbol{a} \in H_{m}(G, \mathbb{Z})$ is said to be reducible if there exists a subgroup $H \varsubsetneqq G$ and a class $\boldsymbol{b} \in H_{m}(H, \mathbb{Z})$ such that $i_{*}(\boldsymbol{b})=\boldsymbol{a}$, where $i$ denotes the canonical inclusion. Otherwise the class will be called irreducible. If the class is reducible then $\mathfrak{S}(G, \boldsymbol{a}) \leq \mathfrak{S}(H, \boldsymbol{b})$ by definition. Now observe that it is trivial to form infinite sequences of classes with bounded systolic volume which reduce to the same class. Indeed consider a homology class $b \in H_{m}(H, \mathbb{Z})$ of dimension $m \geq 2$. Then for any positive integer $n$,

$$
\mathfrak{S}(H * \underbrace{\mathbb{Z} * \cdots * \mathbb{Z}}_{n}, \boldsymbol{a}_{n}) \leq \mathfrak{S}(H, \boldsymbol{b}),
$$

where $\boldsymbol{a}_{n}=\left(i_{n}\right)_{*}(\boldsymbol{b})$ denotes the image of the class $\boldsymbol{b}$ by the natural inclusion $i_{n}: H \hookrightarrow$ $H * \mathbb{Z} * \cdots * \mathbb{Z}$. The existence of infinite sequences of pairwise distinct irreducible classes with bounded systolic volume is much more meaningful and will be proven in Section 4.

Theorem II For any dimension $m \geq 3$ there exists an infinite sequence of finitely presentable pairwise distinct groups $\left\{G_{i}\right\}$ for which at least one irreducible class $\boldsymbol{a}_{i} \in H_{m}\left(G_{i}, \mathbb{Z}\right)$ satisfies $\mathfrak{S}\left(G_{i}, \boldsymbol{a}_{i}\right) \leq 1$.

So we have to introduce some topological or algebraic restrictions in order to get finiteness results. For instance, given a finitely presentable group $G$, a homology class $\boldsymbol{a} \in H_{m}(G, \mathbb{Z})$ and a positive number $T$, the number of integer multiple classes $k \boldsymbol{a}$ whose systolic volume is less than $T$ is at least $T \cdot \ln T$ (up to some multiplicative constant). More precisely, the following theorem will be proven in Section 5.

Theorem III Let $G$ be a finitely presentable group and $\boldsymbol{a} \in H_{m}(G, \mathbb{Z})$, where $m \geq 3$. There exists a positive number $C(G, \boldsymbol{a})$ depending only on the pair $(G, \boldsymbol{a})$ such that

$$
\mathfrak{S}(G, k \boldsymbol{a}) \leq C(G, \boldsymbol{a}) \cdot \frac{k}{\ln (1+k)}
$$


for any positive integer $k$. In particular,

$$
\lim _{k \rightarrow \infty} \frac{\mathfrak{S}(G, k \boldsymbol{a})}{k}=0
$$

This result shows that the systolic volume of multiples of a class is a sublinear function, which is remarkable. For some special classes $\boldsymbol{a}$ (for which simplicial volume is not zero; see Section 5.3), we know after Gromov [17] that there exists a positive number $C^{\prime}(G, \boldsymbol{a})$ depending only on the pair $(G, \boldsymbol{a})$ such that

$$
\mathfrak{S}(G, k \boldsymbol{a}) \geq C^{\prime}(G, \boldsymbol{a}) \cdot \frac{k}{(\ln (1+k))^{m}} .
$$

Moreover, for fundamental groups $\pi_{l}$ of orientable surfaces $\Sigma_{l}$ of genus $l \geq 1$ and for the corresponding fundamental classes $\left[\Sigma_{l}\right]$, we know by [17] and Buser and Sarnak [11] that

$$
\mathfrak{S}\left(\pi_{l}, k\left[\Sigma_{l}\right]\right) \sim \frac{k}{(\ln (1+k))^{2}},
$$

where $f \sim g$ means that there exists some positive constants $c$ and $C$ such that $c \cdot f \leq g \leq C \cdot f$. This naturally leads to the following conjecture.

Conjecture Let $G$ be a finitely presentable group and $a \in H_{m}(G, \mathbb{Z})$ a class of nonzero simplicial volume where $m \geq 3$. Then

$$
\mathfrak{S}(G, k \boldsymbol{a}) \sim \frac{k}{(\ln (1+k))^{m}} .
$$

In Section 6, we explore the case of nilmanifolds, and more specifically the case of the Heisenberg group of dimension 3 . We obtain a new illustration of the possible behavior of the systolic volume of cyclic coverings. The study of the systolic volume of cyclic coverings in terms of the number of sheets has been suggested by Gromov [18], and the first result in this direction can be found in Babenko and Balacheff [5].

The Heisenberg group $\mathcal{H}$ of dimension 3 is the group of triangular matrices

$$
\left\{\left(\begin{array}{lll}
1 & x & z \\
0 & 1 & y \\
0 & 0 & 1
\end{array}\right) \mid x, y, z \in \mathbb{R}\right\} .
$$

The subset $\mathcal{H}(\mathbb{Z})$ of $\mathcal{H}$ composed of matrices with integer coefficients (ie matrices for which $x, y, z \in \mathbb{Z})$ is a lattice, and we will denote by $M_{\mathcal{H}}=\mathcal{H} / \mathcal{H}(\mathbb{Z})$ the corresponding nilmanifold. First of all, we obtain the following explicit upper bound for the systolic volume of multiples of the fundamental class $M_{\mathcal{H}}$. 
Theorem IV Let $\boldsymbol{a}=\left[M_{\mathcal{H}}\right] \in H_{3}(\mathcal{H}(\mathbb{Z}), \mathbb{Z})$ be the fundamental class of $M_{\mathcal{H}}$. Then

$$
\mathfrak{S}(\mathcal{H}(\mathbb{Z}), k \boldsymbol{a}) \leq 19 \cdot \mathfrak{S}(\mathcal{H}(\mathbb{Z}), \boldsymbol{a})
$$

for any integer $k \geq 1$.

The constant appearing here is the one involved in the resolution of the classical Waring problem (see Balasubramanian, Deshouillers and Dress $[7 ; 8]$ ): any integer number decomposes into a sum of at most 19 fourth powers. The idea of using the solution of the Waring problem in order to bound from above the function $\mathfrak{S}(G, k \boldsymbol{a})$ when $(G, \boldsymbol{a})=\left(\mathcal{H}(\mathbb{Z}),\left[M_{\mathcal{H}}\right]\right)$ carries over to any pair $(G, \boldsymbol{a})$, where $G$ is a nilpotent graded group without torsion and $\boldsymbol{a}$ denotes the fundamental class of the corresponding nilmanifold; see Theorem 6.2.

Now consider the sequence of lattices $\left\{\mathcal{H}_{n}(\mathbb{Z})\right\}_{n=1}^{\infty}$ of $\mathcal{H}$, where $\mathcal{H}_{n}(\mathbb{Z})$ denotes the subset of matrices whose integer coefficients satisfy $x \in n \mathbb{Z}$ and $y, z \in \mathbb{Z}$. Denote by $M_{\mathcal{H}_{n}}=\mathcal{H} / \mathcal{H}_{n}(\mathbb{Z})$ the corresponding nilmanifolds. The manifold $M_{\mathcal{H}_{n}}$ is a cyclic covering with $n$ sheets of $M_{\mathcal{H}}$, and the proof of Theorem III implies that

$$
\mathfrak{S}\left(M_{\mathcal{H}_{n}}\right) \leq C \cdot \frac{n}{\ln (1+n)} ;
$$

see Remark 5.5. The fact that the function $\mathfrak{S}\left(M_{\mathcal{H}_{n}}\right)$ goes to infinity is not obvious, and its proof relies on a new invariant for groups called simplicial complexity and defined in Babenko, Balacheff and Bulteau [6]. More precisely, we have the following.

Proposition [6] The function $\mathfrak{S}\left(M_{\mathcal{H}_{n}}\right)$ satisfies the inequality

$$
\mathfrak{S}\left(M_{\mathcal{H}_{n}}\right) \geq a \frac{\ln n}{\exp (b \sqrt{\ln \ln n})},
$$

where $a$ an $b$ are two positive constants.

In particular,

$$
\lim _{n \rightarrow+\infty} \mathfrak{S}\left(M_{\mathcal{H}_{n}}\right)=+\infty .
$$

Note that in this case $\left\|M_{\mathcal{H}_{n}}\right\|_{\Delta}=0$ and the lower bound (1-1) does not apply. For any integer $n$ the manifold $M_{\mathcal{H}_{n}}$ gives a nonnormal realization of the class $n\left[M_{\mathcal{H}}\right]$. So the normalization condition in Theorem I cannot be relaxed.

Acknowledgements The authors would like to thank the anonymous referee for useful comments. This work was partially supported by the grants RFSF 10-01-00257-a and ANR 12-BS01-0009. 


\section{Manifolds with singularities as extrema of the systolic volume}

The systolic volume of a homology class is defined by an infimum. We may wonder if this infimum is reached and what would be the structure of a minimizing pseudomanifold. If the homology class $\boldsymbol{a}$ is realized by a manifold, we know that its systolic volume is a minimum which coincides with the systolic volume of any normal representation of $\boldsymbol{a}$ by a manifold; see $[3 ; 4 ; 10]$. But there exist classes which do not admit representations by manifolds (see [27]), and for such a class $\boldsymbol{a}$ it is not even clear whether $\mathfrak{S}(G, \boldsymbol{a})$ is actually a minimum.

Let $X$ be a pseudomanifold of dimension $m$. The singular locus of $X$ is by definition the set $\Sigma(X)$ of points of $X$ which do not have a neighborhood homeomorphic to an $m$-dimensional ball. By definition of a pseudomanifold, $\Sigma(X)$ is a simplicial subcomplex of codimension at least 2 .

Definition 2.1 A pseudomanifold $X$ is said to be admissible if the natural inclusion $X \backslash \Sigma(X) \subset X$ induces an epimorphism on fundamental groups.

That is, a pseudomanifold is admissible if any element of the fundamental group can be represented by a loop of $X \backslash \Sigma(X)$. A geometric cycle $(X, f)$ representing some homology class $\boldsymbol{a}$ will be called admissible if the pseudomanifold $X$ is admissible.

Example 2.2 Let $M$ be a triangulated manifold and $N \subset M$ be a simplicial subcomplex of codimension greater than or equal to 2 . Denote by $\sharp N$ the set of connected components of $N$. The simplicial complex $M / \sharp N$ obtained from $M$ by contracting the connected components of $N$ into distinct points is an admissible pseudomanifold. The singular locus $\Sigma(M / \sharp N)$ consists of the points corresponding to the connected components of $N$.

Example 2.3 Let $M$ be a manifold with boundary $\partial M=A \times P$, where $A$ is a manifold and $P$ is a connected manifold. The result of the fibred contraction of $P$ is an admissible pseudomanifold $\bar{M}$ homeomorphic to the space

$$
M \cup_{\partial M} A \times C P,
$$

where $C P$ stands for the cone over $P$ and the singular locus $\Sigma(\bar{M})$ is homeomorphic to $A$. Remark that if $P$ is simply connected then $\pi_{1}(\bar{M})=\pi_{1}(M)$. The pseudomanifold $\bar{M}$ obtained that way is a particular example of a singular $P$-manifold; see Baas [1] and Section 2.3 for the general construction. 
Remark that an admissible pseudomanifold of dimension 2 is a surface. In particular it does not possess any singularity.

Theorem I is a direct consequence of the following two propositions.

Proposition 2.4 Let $G$ be a finitely presentable group and $\boldsymbol{a} \in H_{m}(G, \mathbb{Z})$ be a homology class of dimension $m \geq 3$. Suppose that there exists a normal representation of the class $\boldsymbol{a}$ by an admissible geometric cycle $(X, f)$. Then

$$
\mathfrak{S}(G, \boldsymbol{a})=\mathfrak{S}_{f}(X) .
$$

The condition of normalization saying that $f_{\sharp}$ is an epimorphism cannot be dropped; see Section 6 and the example of the Heisenberg group.

The following proposition, together with Proposition 2.4, shows that for any pair $(G, \boldsymbol{a})$ the systolic volume $\mathfrak{S}(G, \boldsymbol{a})$ is actually a minimum.

Proposition 2.5 Let $K$ be a $C W$-complex whose fundamental group is finitely generated. Any homology class $\boldsymbol{a} \in H_{*}(K, \mathbb{Z})$ admits a normal representation by an admissible geometric cycle $(X, f)$.

Before proving Propositions 2.4 and 2.5, we need some technical results.

\subsection{Technical lemmas}

Hopf's trick perfectly adapts to the setting of admissible pseudomanifolds. Consider a map

$$
f:\left(X, X_{1}\right) \rightarrow\left(Y, Y_{1}\right)
$$

between two relative manifolds of the same dimension $m \geq 3$. Suppose that $f$ is transversal at $y \in Y \backslash Y_{1}$, ie there exists an embedded $m$-disk $D$ such that:

- $y \in D \subset Y \backslash Y_{1}$.

- $f^{-1}(D)=\bigcup_{i=1}^{n} D_{i}$ is a disjoint union of $m$-disks embedded in $X \backslash X_{1}$.

- The restriction of $f$ to $f^{-1}(D)$ is a covering map with base space $D$ and $n$ sheets.

Set $x_{i}=D_{i} \cap f^{-1}(y)$. The technical trick by Hopf is essentially contained in the following lemma. 
Lemma 2.6 Suppose that there exists an $m$-disk embedded in $D^{\prime} \subset X \backslash X_{1}$ with the following properties:

(1) $D_{1}, D_{2} \subset D^{\prime}$ and $D^{\prime} \cap D_{i}=\varnothing$ if $i>2$.

(2) For any path $\gamma$ from $x_{1}$ to $x_{2}$ in $D^{\prime}$, the loop $f(\gamma)$ is contractible in $Y$.

(3) For any orientation of $D$, the orientations on $D_{1}$ and $D_{2}$ induced by $f$ are not compatible in $D^{\prime}$.

Then there exists a homotopy $\left\{f_{t}\right\}_{0 \leq t \leq 1}$ of $f_{0}=f$ which is constant on $X \backslash D^{\prime}$ and such that

$$
f_{1}^{-1}(D)=\bigcup_{i>2} D_{i}
$$

the last union being empty if $n=2$.

We refer the reader to Epstein [14, pages 378-380] for a proof of Lemma 2.6, which will work in our setting, as the construction of the homotopy $f_{t}$ occurs in $D^{\prime}$ and therefore carries over to our context. We will now state the corresponding version of Hopf's theorem in the orientable case.

Lemma 2.7 Let $X$ be an admissible orientable connected pseudomanifold and $\left(Y, Y_{1}\right)$ an orientable relative manifold of the same dimension $m \geq 3$. Suppose that $f: X \rightarrow Y$ is a map of degree $k$ inducing an epimorphism between fundamental groups.

Then there exists a homotopy $\left\{f_{t}\right\}_{0 \leq t \leq 1}$ of $f_{0}=f$ such that $f_{1}^{-1}\left(Y \backslash Y_{1}\right)$ is homeomorphic to the disjoint union of $k$ disks and the restriction of $f_{1}$ to the union of these disks is a covering map with base space $Y \backslash Y_{1}$ and $k$ sheets.

The degree of $f$ stands here for the absolute value of the multiple defined by the induced map $f_{*}: H_{m}(X ; \mathbb{Z}) \rightarrow H_{m}\left(Y, Y_{1} ; \mathbb{Z}\right)$. A corresponding version of Lemma 2.7 also holds in the nonorientable context with the notion of absolute degree.

Proof Consider a point $y \in Y \backslash Y_{1}$. We can assume that $y \notin f(\Sigma(X))$ and the map $f$ is transversal at $y$. Let $D \subset Y \backslash Y_{1}$ be a disk containing the point $y$ such that $f^{-1}(D)=\bigcup_{i=1}^{n} D_{i}$ is a disjoint union of $m$-disks embedded in $X \backslash \Sigma(X)$ and such that the restriction of $f$ to $\bigcup_{i=1}^{n} D_{i}$ is a covering map with $n$ sheets and base space $D$. We have $n \geq k$ and suppose that $n>k$. We can choose generators of $H_{m}(X ; \mathbb{Z})$ and $H_{m}\left(Y, Y_{1} ; \mathbb{Z}\right)$ such that the map $f_{*}$ induced on $m$-dimensional homology is simply the multiplication by $k$. This induces an orientation both on $X$ and $Y \backslash Y_{1}$, and also on disks $\left\{D_{i}\right\}_{i=1}^{n}$ and $D$. As $n>k$, there exist two disks, $D_{1}$ and $D_{2}$, such that $\left.f\right|_{D_{1}}$ reverses the orientation and $\left.f\right|_{D_{2}}$ preserves it. We now follow step by step the proof 
of [14, Theorem 4.1]. Join the two points $x_{i}=f^{-1}(y) \cap D_{i}, i=1,2$ by a simple curve $\gamma \subset X \backslash \Sigma(X)$. Because $f$ induces an epimorphism between fundamental groups, there exists a loop $\alpha$ based at $x_{1}$ such that $f(\alpha)$ and $f(\gamma)$ are homotopic as loops based at $y$. As $X$ is admissible, we can furthermore choose $\alpha$ in $X \backslash \Sigma(X)$. The concatenation $\alpha^{-1} \star \gamma$ and its evident modification in a neighborhood of the concatenation define a simple curve $\beta \subset X \backslash \Sigma(X)$ joining $x_{1}$ and $x_{2}$. The loop $f(\beta)$ is contractible in $Y$ relatively to $y$. We then define the disk $C$ as a small enough neighborhood of $\beta$, and apply Lemma 2.6. Remark that the choice of $C$ implies a possible diminution of the size of the disks $\left\{D_{i}\right\}_{i=1}^{n}$ and $D$. The end of the proof is straightforward; see [14] for the missing details.

\subsection{Admissible geometric cycles are minima of the systolic volume}

In this subsection, we prove Proposition 2.4. For this we use in a decisive way the comparison and extension techniques elaborated in [3], as well as the ideas contained in this article. For the reader's convenience, let first recall these two techniques.

We first present the comparison principle which originally appeared as [3, Proposition 3.2]. Given two pseudomanifolds $X_{1}$ and $X_{2}$ recall that a simplicial map from $X_{1}$ to $X_{2}$ is called $m$-monotone if the preimage of any open $m$-simplex is either an open $m$-simplex or empty.

Proposition 2.8 (Comparison principle) Let $\left(X_{1}, f_{1}\right)$ and $\left(X_{2}, f_{2}\right)$ be two geometric cycles representing an homology class $\boldsymbol{a} \in H_{m}(G, \mathbb{Z})$ and suppose that there exists an $m$-monotone simplicial map $p: X_{1} \rightarrow X_{2}$ such that $f_{1}=f_{2} \circ p$. Then

$$
\mathfrak{S}_{f_{1}}\left(X_{1}\right) \leq \mathfrak{S}_{f_{2}}\left(X_{2}\right)
$$

Now we state the extension principle; see [3, Proposition 3.6].

Proposition 2.9 (Extension principle) Let $\left(X_{1}, f_{1}\right)$ be a geometric cycle representing a homology class of dimension $m$. Suppose that $X_{2}$ is a $C W$-complex obtained from $X_{1}$ by adding a finite number of cells with dimension from 3 to $m-1$. Then

$$
\mathfrak{S}_{f_{1}}\left(X_{1}\right)=\mathfrak{S}_{f_{2}}\left(X_{2}\right) \text {, }
$$

where $f_{2}: X_{2} \rightarrow K(G, 1)$ is the unique (up to homotopy) extension of $f_{1}$.

We now present the proof of Proposition 2.4.

Let $\left(X_{1}, f_{1}\right)$ be a geometric cycle representing the class $\boldsymbol{a}$. The pseudomanifold $X_{1}$ admits a cell decomposition with only one $m$-cell (see for example Sabourau [24]). 
This allows us to describe $X_{1}$ as a relative $m$-manifold $\left(Y, Y^{\prime}\right)$, where $Y$ denotes the $m$-cell and $Y^{\prime}$ lies in the $(m-1)$-skeleton. Following [3] we construct an extension of $X_{1}$ as follows.

We start by adding a finite number of $1-$ and 2-cells to $X_{1}$ such that the resulting CW-complex $X_{1}(2)$ has fundamental group $G$ and the inclusion map $i: X_{1} \hookrightarrow X_{1}(2)$ satisfies $i_{\sharp}=\left(f_{1}\right)_{\sharp}$. More precisely, given a finitely generated presentation of $G$ we first construct a 2-dimensional finite cellular complex $K^{(2)}$ by considering the wedge sum of 1 -cells corresponding to the generators of the presentation of $G$, and then gluing 2-cells according to its relations. The finite cellular complex $X(2)$ is then obtained as the wedge sum of $X_{1}$ and $K^{(2)}$, with additional 2-cells glued along the image of $\left(f_{1}\right)_{\sharp}\left(\gamma_{i}\right) * \gamma_{i}^{-1}$ for a finite set $\left\{\gamma_{i}\right\}_{i \in I}$ of generators of $\pi_{1}\left(X_{1}\right)$.

Lemma 2.10 We have

$$
\mathfrak{S}_{f_{1}}\left(X_{1}\right)=\mathfrak{S}(X(2)) \text {. }
$$

Proof In fact, using the comparison principle, we first observe that

$$
\mathfrak{S}_{f_{1}}\left(X_{1}\right) \leq \mathfrak{S}\left(X_{1}(2)\right) \text {. }
$$

Now fix a positive $\varepsilon$ and a metric $g_{1}$ on $X_{1}$ such that

$$
\frac{\operatorname{vol}\left(X_{1}, g_{1}\right)}{\operatorname{sys}_{f_{1}}\left(X_{1}, g_{1}\right)^{m}} \leq \mathfrak{S}_{f_{1}}\left(X_{1}\right)+\varepsilon .
$$

Now define the metric $g$ on $X_{1}(2)$ which coincides with $g_{1}$ on $X_{1}$, for which the length of each additional 1 -cell is exactly $\operatorname{sys}_{f_{1}}\left(X_{1}, g_{1}\right)$ and for which each additional $2-$ cell is a round hemisphere whose curvature is chosen accordingly to the length of its boundary. It is straightforward to see that any closed curve of $X_{1}(2)$ can be homotoped to a curve lying in the union of $X_{1}$ and the 1 -skeleton of $K^{(2)}$ without increasing the length. In particular sys $\left(X_{1}(2), g\right)=\operatorname{sys}_{f_{1}}\left(X_{1}, g_{1}\right)$. But $\operatorname{vol}\left(X_{1}(2), g\right)=\operatorname{vol}\left(X_{1}, g_{1}\right)$ for dimensional reasons as $m \geq 3$ so we get

$$
\mathfrak{S}\left(X_{1}(2)\right) \leq \frac{\operatorname{vol}\left(X_{1}(2), g\right)}{\operatorname{sys}\left(X_{1}(2), g\right)^{m}} \leq \mathfrak{S}_{f_{1}}\left(X_{1}\right)+\varepsilon,
$$

which concludes the proof.

Then, for each dimension $k$ going from 3 to $m$, we add $k$-cells to $X_{1}(k-1)$ such that the new CW-complex $X_{1}(k)$ thus obtained satisfies $\pi_{i}\left(X_{1}(k)\right)=0$ for $1<i<k-1$. At the end we get a CW-complex denoted by $X_{1}(m)$ which is $m$-aspherical and whose fundamental group is $G$. Remark that $Y \backslash Y^{\prime}$ is an $m$-cell of $X_{1}(m)$. By adding cells of dimension higher than $m$, we can realize the Eilenberg-Mac Lane space $K(G, 1)$ as an extension of $X_{1}(m)$. 
By assumption there exists an admissible pseudomanifold $X$ and a map $f: X \rightarrow$ $K(G, 1)$ giving a realization of the class $\boldsymbol{a} \in H_{m}(G, \mathbb{Z})$ such that $f_{\sharp}: \pi_{1}(X) \rightarrow G$ is an epimorphism. By the cellular approximation theorem and according to [3, Lemma 3.10] which applies to this situation, we can find a map

$$
g: X \rightarrow X_{1}(m) \subset K(G, 1)
$$

homotopic to $f$ in $K(G, 1)$ such that

$$
g_{*}[X]=i_{*}\left[X_{1}\right]
$$

in $H_{m}\left(X_{1}(m), \mathbb{Z}\right)$. Since $g$ is homotopic to $f$, the induced map $g_{\sharp}$ is still an epimorphism and

$$
\mathfrak{S}_{f}(X)=\mathfrak{S}_{g}(X) .
$$

Let $\{Y\} \cup\left\{Y_{i}\right\}_{i \in I}$ denote the $m$-cells of $X_{1}(m)$ (this list can be finite or infinite). To each $m$-cell $Y_{i}$ or $Y$ is associated the relative manifold $\left(Y_{i}, \hat{Y}_{i}\right)$ or $(Y, \hat{Y})$, where $\hat{Y}_{*}$ denotes the closure of the union of all the other cells of $Y_{*}$. The map $g$ induces maps

$$
\tilde{g}: X \rightarrow(Y, \hat{Y}) \quad \text { and } \quad \tilde{g}_{i}: X \rightarrow\left(Y_{i}, \hat{Y}_{i}\right) \quad \text { for all } i \in I,
$$

which still induce epimorphisms between fundamental groups. From (2-1), we deduce that the degree of $\tilde{g}$ is equal to 1 , and that the degree of each $\tilde{g}_{i}$ is 0 . As $X$ is compact, $g(X)$ intersects only a finite number of $m$-cells. We then apply Lemma 2.7 to each relative manifold $\left(Y_{i}, \hat{Y}_{i}\right)$ such that $Y_{i} \cap g(X) \neq \varnothing$. In this way we obtain a map

$$
g_{1}: X \rightarrow Z \subset X_{1}(m-1)
$$

homotopic to $g$ where $Z$ denotes the subcomplex of $X_{1}(m-1)$ obtained from $X_{1}(2)$ by adding the cells with dimension from 3 to $m-1$ which intersect $g_{1}(X)$. Observe that these cells are in finite number so

$$
\mathfrak{S}(Z)=\mathfrak{S}\left(X_{1}(2)\right)
$$

according to the extension principle. The map can be chosen to be $m$-monotone and simplicial; see [3, Proposition 3.13]. Thus

$$
\mathfrak{S}_{g}(X)=\mathfrak{S}_{g_{1}}(X) \leq \mathfrak{S}(Z)
$$

according to the comparison principle.

To summarize, we have shown that

$$
\mathfrak{S}_{f}(X) \leq \mathfrak{S}_{f_{1}}\left(X_{1}\right)
$$


for any representation $\left(X_{1}, f_{1}\right)$ of $\boldsymbol{a}$ which proves that

$$
\mathfrak{S}_{f}(X)=\mathfrak{S}(G, \boldsymbol{a}) .
$$

\subsection{Manifolds with singularities of prescribed type according to Baas}

In order to prove Proposition 2.5, we briefly recall a type of singular manifolds introduced by $\mathrm{N}$ Baas. We follow the presentation of [1].

First recall the following definition due to J Cerf; see [12].

Definition 2.11 A manifold with general corners of dimension $m$ is a Hausdorff space locally modeled on the $m$-cube $[0,1]^{m}$.

In other words, manifolds with general corners are manifolds whose boundary looks like the boundary of the cube. A manifold with general corners can be smoothed through a process which has been well studied (see [12] for instance).

To define manifolds with reasonable singularities, N Baas - following D Sullivan's idea-considers manifolds with general corners whose boundary decomposes as products and contracts some terms of these products, like in Example 2.3. To describe his construction let us recall the following definition.

Definition 2.12 A manifold $M^{m}$ with general corners is said to be decomposed if its boundary decomposes into a union

$$
\partial M=\partial_{0} M \cup \partial_{1} M \cup \cdots \cup \partial_{n} M
$$

of codimension- 1 submanifolds with general corners. Here the union means the identification along a common part of the boundaries of the $\partial_{i} M$.

If $M$ is a decomposed manifold, each of its boundary terms turns out to be a decomposed manifold too. More precisely, by setting

$$
\left\{\begin{array}{l}
\partial_{j}\left(\partial_{i} M\right)=\partial_{j} M \cap \partial_{i} M \quad \text { if } j \neq i, \\
\partial_{i}\left(\partial_{i} M\right)=\varnothing
\end{array}\right.
$$

we get

$$
\partial\left(\partial_{i} M\right)=\bigcup_{j=0}^{n} \partial_{j}\left(\partial_{i} M\right)
$$

and each $\partial_{i} M$ is again a decomposed manifold. 
Example 2.13 If $M$ denotes the $m$-dimensional cube, its boundary is naturally decomposed into $(m-1)$-faces:

$$
\partial M=\partial_{0} M \cup \partial_{1} M \cup \cdots \cup \partial_{2 m-1} M .
$$

We consider a finite sequence of closed manifolds $S=\left\{P_{0}=*, P_{1}, \ldots, P_{n}\right\}$ ordered by increasing dimension. These manifolds will play the role of "singularities" after their contraction. Before the contraction process, the following definition describes how the manifolds should look in order to prescribe the type of singularities we will obtain.

Definition 2.14 A manifold $M$ with general corners is said to be an $S$-manifold if:

(1) For any subset $\omega \subset\{0,1, \ldots, n\}$, there exists a decomposed manifold $M(\omega)$ such that:

(a) $M(\varnothing)=M$.

(b) $\partial M(\omega)=\bigcup_{i \notin \omega} \partial_{i} M(\omega)$.

(c) For every $i \in\{1, \ldots, n\} \backslash \omega$, there exists a diffeomorphism

$$
\beta(\omega, i): \partial_{i} M(\omega) \simeq M(\omega, i) \times P_{i},
$$

where $(\omega, i)=\omega \cup\{i\}$.

(2) For any subset $\omega \subset\{0,1, \ldots, n\}$, and for all $i, j \in\{1, \ldots, n\} \backslash \omega$, the diagram

$$
\begin{aligned}
& \partial_{j} \partial_{i} M(\omega) \stackrel{\beta(\omega, i)}{\|} \partial_{j} M(\omega, i) \times P_{i} \stackrel{\beta(\omega, i, j) \times \mathrm{id}}{\longrightarrow} M(\omega, i, j) \times P_{j} \times P_{i} \\
& \partial_{j} M(\omega) \cap \partial_{i} M(\omega) \\
& \| \downarrow_{\mathrm{id} \times T} \\
& \partial_{i} \partial_{j} M(\omega) \stackrel{\beta(\omega, j)}{\longrightarrow} \partial_{j} M(\omega, i) \times P_{j} \stackrel{\beta(\omega, j, i) \times \text { id }}{\longrightarrow} M(\omega, i, j) \times P_{i} \times P_{j}
\end{aligned}
$$

is commutative, where $T$ denotes the transposition.

The first part of the definition describes the local structure of the product on the boundary of the decomposed manifold $M$. The diagram describes how the boundary components are glued together. We now define the particular class of singular manifolds pointed out by N Baas.

Definition 2.15 To any $S$-manifold $M$ we associate the singular manifold $M_{S}$ defined as the quotient space $M(\varnothing) / \sim$, where

$$
a \sim b \quad \text { if } \quad a, b \in \partial_{i_{1}} \cdots \partial_{i_{k}} M, i_{j} \geq 1, k \geq 1,
$$


and

$$
\begin{aligned}
\operatorname{pr} \circ \beta\left(i_{1}, \ldots, i_{k}\right) \circ \cdots \circ \beta\left(i_{1}, i_{2}\right) \circ \beta\left(\varnothing, i_{1}\right)(a) \\
=\operatorname{pr} \circ \beta\left(i_{1}, \ldots, i_{k}\right) \circ \cdots \circ \beta\left(i_{1}, i_{2}\right) \circ \beta\left(\varnothing, i_{1}\right)(b) .
\end{aligned}
$$

Here

$$
\text { pr: } M\left(i_{1}, \ldots, i_{k}\right) \times P_{i_{1}} \times \cdots \times P_{i_{k}} \rightarrow M\left(i_{1}, \ldots, i_{k}\right)
$$

denotes projection on the first factor.

If the elements of $S$ are connected manifolds, then every manifold $M$ with singularities of type $S$ is an admissible pseudomanifold. If not, the following remark will be of fundamental importance in the next section.

Remark 2.16 For each $i=1, \ldots, n$, we decompose the manifold $P_{i}$ into connected components $Q_{i j}$ and set

$$
T=\left\{Q_{i j} \mid 1 \leq i \leq n, 1 \leq j \leq k_{i}\right\} .
$$

Given a manifold $M_{S}$ with singularities of type $S$ modeled on $M$, the local $S-$ structure (2-3) on $\partial M$ defines a local $T$-structure. The commutative diagram of Definition 2.14 and the equivalence (2-4) allow us to define an equivalence relation on $M$ denoted by $\sim_{T}$ and such that the projections (2-5) only occur along the factors of type $Q_{i j}$. This gives rise to a $T$-singular manifold $M_{T}$ defined as the quotient $M(\varnothing) / \sim_{T}$. A class for the relation $\sim_{T}$ being a subclass of the relation $\sim_{S}$, we get a canonical map of degree 1 :

$$
q: M_{T} \rightarrow M_{S}
$$

\subsection{Realization of homology classes by admissible geometric cycles}

We now prove Proposition 2.5. Following Milnor [21] and Novikov [22], the complex cobordism ring $\Omega_{*}^{U}$ is isomorphic to the ring of integer polynomials $\Omega_{*}^{U}=$ $\mathbb{Z}\left[x_{1}, x_{2}, \ldots\right]$, where each generator $x_{k}$ is of degree $2 k$ and can be represented by a manifold $P_{k}$. Each representant $P_{k}$ can be chosen as a complex algebraic manifold; see Stong [26] for instance. But the connectivity of this complex manifold is not clear in general (if $k=p^{s}-1$, where $p$ is a prime number, $P_{k}$ can be chosen as $\mathbb{C} P^{k}$ ). Define the sequence of singularities

$$
S=\left\{P_{1}, P_{2}, \ldots\right\} .
$$

According to Baas' theorem [1, Corollaire 5.1], given a homology class $\boldsymbol{a} \in H_{m}(X, \mathbb{Z})$, there exists a manifold $M_{S}$ with singularities of type $S$ of dimension $m$ and a map 
$f: M_{S} \rightarrow X$ such that $f_{*}\left[M_{S}\right]=\boldsymbol{a}$. The elements in $S$ are not necessarily connected manifolds. So we proceed as in Remark 2.16, and obtain in this way a new manifold with singularities $M^{\prime}$ representing $\boldsymbol{a}$ which is now an admissible pseudomanifold. Finally, we add if necessary 1-handles to $M^{\prime}$ and extend the map $f^{\prime}=f \circ q$ (where $q$ denotes the canonical map from $M^{\prime}$ to $M_{S}$ of degree 1 ) in such a way that $f_{\sharp}^{\prime}$ becomes an epimorphism between fundamental groups. This concludes the proof.

Remark 2.17 The admissible pseudomanifold $M^{\prime}$ which realizes $\boldsymbol{a}$ can be chosen as a manifold with singularities whose singularities are more specific. In $\mathbb{C} P^{m} \times \mathbb{C} P^{n}$ with $m \leq n$, we consider the hypersurface of degree $(1,1)$ given by

$$
H_{m, n}=\left\{z_{0} w_{0}+z_{1} w_{1}+\cdots+z_{m} w_{m}=0\right\},
$$

where $\left(z_{0}, z_{1}, \ldots, z_{m}\right)$ and $\left(w_{0}, w_{1}, \ldots, w_{n}\right)$ denote the homogeneous coordinates in $\mathbb{C} P^{m}$ and $\mathbb{C} P^{n}$ respectively. The manifolds $H_{m, n}$ are known as Milnor's manifolds. The cobordism classes of the $\left\{H_{m, n}\right\}_{m \leq n}$ together with the family of classes $\left\{\mathbb{C} P^{s}\right\}_{s \geq 1}$ give rise to a spanning family of $\Omega_{*}^{U}$ (see Hirzebruch [19] and [22]). The classes in $\Omega_{*}^{U}$ are thus linear combinations (with integer coefficients) of cobordism classes $\left\{H_{m, n}\right\}_{m \leq n}$ and $\left\{\mathbb{C} P^{s}\right\}_{s \geq 1}$. So we can choose the $\left\{P_{k}\right\}_{k \geq 1}$ as a disjoint union of some of these manifolds endowed with an adequate orientation. Taking into account Remark 2.16, an admissible pseudomanifold which represents the class $a \in H_{*}(K, \mathbb{Z})$ can be chosen as a manifold with singularities of type $\left\{\mathbb{C} P^{s}, H_{m, n}\right\}$.

\section{Relative density of the values of systolic volume}

The aim of this section is to show that the set of values of systolic volume over the set of homology classes (resp. over the set of orientable manifolds) of fixed dimension is a relatively dense set in the following sense.

Definition 3.1 Given a subset $A \subset \mathbb{R}^{+}$and a positive number $d, A$ is said to be $d$-dense in $\mathbb{R}^{+}$if for any interval $I \subset \mathbb{R}^{+}$of length $|I|>d$, the intersection $I \cap A$ is not empty.

For a fixed dimension $m \geq 3$, define

$\Sigma_{m}:=\left\{\mathfrak{S}(G, \boldsymbol{a}) \mid G\right.$ finitely presentable group and $\left.\boldsymbol{a} \neq 0 \in H_{m}(G, \mathbb{Z})\right\}$,

$$
\sigma_{m}:=\inf \Sigma_{m}
$$

Similarly, set

$$
\begin{gathered}
\Omega_{m}^{+}:=\{\mathfrak{S}(M) \mid M \text { orientable essential manifold of dimension } m\}, \\
\omega_{m}^{+}:=\inf \Omega_{m}^{+} .
\end{gathered}
$$


Recall that $\sigma_{m}>0$ by [17] and that an orientable manifold $M$ is said to be essential if the image of its fundamental class under its classifying map is not zero. In particular, $\omega_{m}^{+} \geq \sigma_{m}$. The main result of this section is the following proposition.

Proposition 3.2 For any dimension $m \geq 3$, the set $\Sigma_{m}$ (resp. $\Omega_{m}^{+}$) is $\sigma_{m}$-dense (resp. $\omega_{m}^{+}$-dense) in $\mathbb{R}^{+}$.

Proposition I of the introduction is a direct consequence of this statement. In order to prove Proposition 3.2, we will study the behavior of systolic volume under the operation of connected sum. Part of these results will also be useful in the next section.

Remark 3.3 Remark that the set $\Omega_{m}$ of values taken by the systolic volume over all manifolds of the same dimension $m$ (not necessarily orientable) contains the subset $\Omega_{m}^{+}$: it is also a relatively dense set of $\mathbb{R}^{+}$with density $\omega_{m}^{+}$. It is not clear if this density can be decreased and the answer may depend on the parity of the dimension.

Fix a morphism of groups $\pi: G \rightarrow G^{\prime}$ and a homology class $\boldsymbol{a} \in H_{m}(G, \mathbb{Z})$.

Proposition 3.4 If $m \geq 3$,

$$
\mathfrak{S}\left(G^{\prime}, \pi_{*} \boldsymbol{a}\right) \leq \mathfrak{S}(G, \boldsymbol{a}) .
$$

Proof According to Proposition 2.5, fix an admissible geometric cycle $(X, f)$ representing normally the class $\boldsymbol{a}$. The admissible geometric cycle $(X, \pi \circ f)$ is a representation of the class $\pi_{*}(\boldsymbol{a})$, so

$$
\mathfrak{S}\left(G^{\prime}, \pi_{*}(\boldsymbol{a})\right) \leq \mathfrak{S}_{\pi \circ f}(X) \leq \mathfrak{S}_{f}(X)=\mathfrak{S}(G, \boldsymbol{a})
$$

by Proposition 2.4 .

Corollary 3.5 Let $X_{1}$ and $X_{2}$ be two orientable admissible pseudomanifolds of dimension $m \geq 3$. Then

$$
\max \left\{\mathfrak{S}\left(X_{1}\right), \mathfrak{S}\left(X_{2}\right)\right\} \leq \mathfrak{S}\left(X_{1} \# X_{2}\right),
$$

where $X_{1} \# X_{2}$ denotes the connected sum of $X_{1}$ and $X_{2}$.

Proof Denote by $f_{j}: X_{j} \rightarrow K\left(\pi_{1}\left(X_{j}\right), 1\right)$ the classifying map for $j=1,2$. Observe that $\pi_{1}\left(X_{1} \# X_{2}\right)=\pi_{1}\left(X_{1}\right) * \pi_{1}\left(X_{2}\right)$. We have a natural monomorphism $i_{j}: \pi_{1}\left(X_{j}\right) \hookrightarrow$ 
$\pi_{1}\left(X_{1}\right) * \pi_{1}\left(X_{2}\right)$ and a natural epimorphism $s_{j}: \pi_{1}\left(X_{1}\right) * \pi_{1}\left(X_{2}\right) \rightarrow \pi_{1}\left(X_{j}\right)$ such that $s_{j} \circ i_{j}=\mathrm{id}_{\pi_{1}\left(X_{j}\right)}, s_{2} \circ i_{1}=0$ and $s_{1} \circ i_{2}=0$. By Proposition 3.4,

$$
\begin{aligned}
\mathfrak{S}\left(X_{1} \# X_{2}\right) & =\mathfrak{S}\left(\pi_{1}\left(X_{1}\right) * \pi_{1}\left(X_{2}\right),\left(i_{1} \circ f_{1}\right)_{*}\left[X_{1}\right]+\left(i_{2} \circ f_{2}\right)_{*}\left[X_{2}\right]\right) \\
& \geq \mathfrak{S}\left(s_{j}\left(\pi_{1}\left(X_{1}\right) * \pi_{1}\left(X_{2}\right)\right),\left(s_{j}\right)_{*} \circ\left(\left(i_{1} \circ f_{1}\right)_{*}\left[X_{1}\right]+\left(i_{2} \circ f_{2}\right)_{*}\left[X_{2}\right]\right)\right) \\
& \left.\geq \mathfrak{S}\left(\pi_{1}\left(X_{j}\right),\left(f_{j}\right)_{*}\left[X_{j}\right]\right)\right\}=\mathfrak{S}\left(X_{j}\right)
\end{aligned}
$$

for $j=1,2$.

Furthermore, we have the following comparison result:

Proposition 3.6 Let $X_{1}$ and $X_{2}$ be two orientable pseudomanifolds of dimension $m \geq 3$. Then

$$
\mathfrak{S}\left(X_{1} \# X_{2}\right) \leq \mathfrak{S}\left(X_{1}\right)+\mathfrak{S}\left(X_{2}\right)
$$

Proof The contraction of the gluing sphere into a point gives rise to a natural projection map

$$
p: X_{1} \# X_{2} \rightarrow X_{1} \vee X_{2}
$$

which induces an isomorphism between fundamental groups if $m \geq 3$. Applying the comparison principle (see [3, Proposition 3.2]), we get

$$
\mathfrak{S}\left(X_{1} \# X_{2}\right) \leq \mathfrak{S}\left(X_{1} \vee X_{2}\right)=\mathfrak{S}\left(X_{1}\right)+\mathfrak{S}\left(X_{2}\right) .
$$

With these two comparison results, we can now prove Proposition 3.2.

We first prove the $\sigma_{m}$-density of the set $\Sigma_{m}$. Consider any interval $I \subset \mathbb{R}^{+}$such that $|I|>\sigma_{m}$. Choose a pair $(G, \boldsymbol{a})$ composed of a finitely presentable group and an $m$-class of its homology such that $\sigma_{m} \leq \mathfrak{S}(G, \boldsymbol{a})<|I|$. According to Proposition 2.5, we can choose an admissible geometric cycle $(X, f)$ representing normally the class $\boldsymbol{a} \in H_{m}(G, \mathbb{Z})$ which satisfies $\mathfrak{S}_{f}(X)=\mathfrak{S}(G, \boldsymbol{a})$ by Proposition 2.4. If the map $f_{\sharp}$ is not a monomorphism, we can directly contract some loops in $X$ and get a new admissible geometric cycle $\left(X^{\prime}, f^{\prime}\right)$ representing $\boldsymbol{a}$ such that $f_{\sharp}^{\prime}$ is an isomorphism. So

$$
\mathfrak{S}_{f}(X)=\mathfrak{S}\left(X^{\prime}\right) .
$$

The sequence $\left\{a_{n}=\mathfrak{S}\left(\#_{n} X^{\prime}\right)\right\}_{n=1}^{\infty}$ is increasing by Corollary 3.5 and satisfies $a_{n+1}-$ $a_{n} \leq \mathfrak{S}\left(X^{\prime}\right)<|I|$ by Proposition 3.6. By Sabourau [25], we have

$$
\mathfrak{S}(\underset{n}{\#} X) \geq C_{m} \frac{n}{\exp \left(C_{m}^{\prime} \sqrt{\log n}\right)},
$$


where $C_{m}$ and $C_{m}^{\prime}$ are two positive numbers depending only on the dimension $m$ (strictly speaking, this inequality is proved for manifolds, but carries over perfectly to pseudomanifolds). So the sequence $\left\{a_{n}\right\}$ is not bounded and does intersect $I$.

The proof of the $\omega_{m}^{+}$-density of $\Omega_{m}^{+}$is similar: for any interval $I \subset \mathbb{R}^{+}$with $|I|>\omega_{m}^{+}$, we can argue as previously starting with the sequence $\left\{a_{n}=\mathfrak{S}\left(\#_{n} M\right)\right\}_{n=1}^{\infty}$, where $M$ is an orientable essential manifold of dimension $m$ with $\omega_{m}^{+} \leq \mathfrak{S}(M)<|I|$.

\section{Nonfiniteness of irreducible homology classes}

This section deals with the following natural question:

Question 4.1 Given a positive number $T$, how many finitely presentable groups $G$ are there, such that any essential (orientable) manifold $M$ of dimension $m$ with fundamental group $G$ satisfies $\mathfrak{S}(M) \leq T$ ?

In dimension 2 , this number is bounded from below by $c \cdot T(\ln T)^{2}$ and from above by $C \cdot T(\ln T)^{2}$ for some positive constants $c$ and $C$; see $[11 ; 18]$. In fact the situation is quite rigid in dimension 2 . Even the finiteness of the systolic volume over the set of finite simplicial complexes of dimension 2 holds. More precisely, recall that:

- The systolic area of a finitely presentable group $G$ is defined as

$$
\mathfrak{S}(G)=\inf _{\pi_{1}(P)=G} \mathfrak{S}(P),
$$

where the infimum is taken over all finite simplicial complexes $P$ of dimension 2 with fundamental group $G$.

- A finitely presentable group $G$ is said to be of zero free index if $G$ cannot be written as a free product $H * F_{n}$ for some $n>0$; compare Rudyak and Sabourau [23].

Then the number of finitely presentable groups $G$ of zero free index such that $\mathfrak{S}(G) \leq T$ does not exceed $K^{T^{3}}$ where $K>1$ is an explicit constant; see [23].

The situation is rather different in higher dimensions. Let $M$ be an essential manifold of dimension $m \geq 4$ whose fundamental group is of zero free index and $N$ a nonessential manifold of the same dimension with fundamental group of zero free index. The fundamental group $\pi_{1}(M) * \pi_{1}(N)$ of the connected sum $M \# N$ is still of zero free index. By Proposition 3.6 we get

$$
\mathfrak{S}(M \# N) \leq \mathfrak{S}(M) .
$$


(Recall that nonessential orientable manifolds have systolic volume equal to zero; see Babenko [2] for instance). That is, while staying in the class of groups of zero free index we can considerably modify the fundamental group of a manifold without increasing the systolic volume. So there is no hope to obtain finiteness results in this context. This is why we introduce the following.

Definition 4.2 Let $G$ be a finitely presentable group. A class $\boldsymbol{a} \in H_{m}(G, \mathbb{Z})$ is said to be reducible if there exists a proper subgroup $H<G$ and a class $\boldsymbol{b} \in H_{m}(H, \mathbb{Z})$ such that $i_{*}(\boldsymbol{b})=\boldsymbol{a}$, where $i$ denotes the canonical inclusion. In the contrary case, the class $\boldsymbol{a}$ is said to be irreducible.

Furthermore, we will say that a manifold $M$ is reducible (resp. irreducible) if the image of its fundamental class $[M]$ (under the classifying map) in $H_{m}\left(\pi_{1}(M), \mathbb{Z}\right)$ is a reducible (resp. irreducible) class.

Example 4.3 Let $M$ be an aspherical manifold of dimension $m$ (that is $\pi_{k}(M)=0$ for $k>1)$. Then $M$ is irreducible.

On the other hand if $M$ and $N$ are nonsimply connected manifolds and $N$ is not essential then the connected sum $M \# N$ is always reducible.

Example 4.4 Let $G$ be a finite group and $\boldsymbol{a} \in H_{m}(G, \mathbb{Z})$ be a class of order $|G|$. Then $\boldsymbol{a}$ is irreducible.

This last example shows that the fundamental class of a lens manifold is irreducible.

Remark that it is possible that:

- Any multiple of an irreducible class is irreducible as in the case $G=\mathbb{Z}_{p}$ for $p$ a prime number.

- Each multiple of an irreducible class is reducible as in the case of tori $\mathbb{T}^{m}$.

On the other hand, there exist groups $G$ and classes $\boldsymbol{a} \in H_{m}(G, \mathbb{Z})$ which are completely reducible in the following sense: $\boldsymbol{a}$ is reducible, and any class $\boldsymbol{b} \in H_{m}(H, \mathbb{Z})$, where $H$ is a proper subgroup $H \subset G$ and such that $i_{*}(\boldsymbol{b})=\boldsymbol{a}$ is also reducible.

Given a positive number $T$ and a positive integer $m$, we denote by $\mathcal{F}(m, T)$ the set of finitely presentable groups $G$ such that there exists an irreducible class $\boldsymbol{a} \in H_{m}(G, \mathbb{Z})$ with $\mathfrak{S}(\boldsymbol{a}) \leq T$. Theorem II of the introduction can be now restated as follows.

Theorem 4.5 The set $\mathcal{F}(m, 1)$ is infinite for any dimension $m \geq 3$. 
Proof Let $p$ be a prime number and set

$$
G(p, m):=\underbrace{\mathbb{Z}_{p} \oplus \cdots \oplus \mathbb{Z}_{p}}_{m} .
$$

Denote by $\phi_{p}: \pi_{1}\left(\mathbb{T}^{m}\right) \rightarrow G(p, m)$ the natural projection and set

$$
\boldsymbol{a}(p, m):=\left(\phi_{p}\right)_{*}\left[\mathbb{T}^{m}\right] \in H_{m}(G(p, m), \mathbb{Z}) .
$$

In order to prove that $\boldsymbol{a}(p, m) \neq 0$ in $H_{m}(G(p, m), \mathbb{Z})$, we will show that the reduction modulo $p$ of $\boldsymbol{a}(p, m)$ is not null in $H_{m}\left(G(p, m), \mathbb{Z}_{p}\right)$. Consider the generators $v_{1}, \ldots, v_{m}$ of $H^{1}\left(G(p, m), \mathbb{Z}_{p}\right)$ corresponding to the natural projections of $G(p, m)$ on each factor. The elements $u_{i}:=\left(\phi_{p}\right)^{*}\left(v_{i}\right), i=1,2, \ldots, m$ generate the group $H^{1}\left(\mathbb{T}^{m}, \mathbb{Z}_{p}\right)$, and $u:=u_{1} \cup \cdots \cup u_{m}$ generates the group $H^{m}\left(\mathbb{T}^{m}, \mathbb{Z}_{p}\right)$. So $u \cap\left[\mathbb{T}^{m}\right]_{p}=1$, where $\left[\mathbb{T}^{m}\right]_{p}$ denotes the reduction modulo $p$ of the fundamental class $\left[\mathbb{T}^{m}\right]$. This implies

$$
\left(v_{1} \cup \cdots \cup v_{m}\right) \cap\left(\phi_{p}\right)_{*}\left[\mathbb{T}^{m}\right]_{p}=\left(\phi_{p}\right)^{*}\left(v_{1}\right) \cup \cdots \cup\left(\phi_{p}\right)^{*}\left(v_{m}\right) \cap\left[\mathbb{T}^{m}\right]_{p}=1 .
$$

This proves the nontriviality of $\left(\phi_{p}\right)_{*}\left[\mathbb{T}^{m}\right]_{p}$, and thus of $\left(\phi_{p}\right)_{*}\left[\mathbb{T}^{m}\right]$.

We now prove the irreducibility of $\boldsymbol{a}(p, m)$. Let us suppose the contrary. Any proper subgroup $H$ of $G(p, m)$ is also a $\mathbb{Z}_{p}$-vector subspace of $G(p, m)$ of dimension $k<m$. Associated to some complement of $H$ in $G(p, m)$, we construct a projection map

$$
\pi: G(p, m) \rightarrow H
$$

which is the identity on $H$. Fix a basis of the free $\mathbb{Z}$-module $\pi_{1}\left(\mathbb{T}^{m}\right)$ such that the composition

$$
\pi \circ \phi_{p}: \pi_{1}\left(\mathbb{T}^{m}\right) \rightarrow H
$$

decomposes as

$$
\pi \circ \phi_{p}=\psi \circ \rho_{\sharp},
$$

where $\rho_{\sharp}$ is induced by some projection $\rho: \mathbb{T}^{m} \rightarrow \mathbb{T}^{k}$ and $\psi: \pi_{1}\left(\mathbb{T}^{k}\right) \rightarrow H$ corresponds to the reduction modulo $p$. Now assume that $\boldsymbol{a}(p, m)=i_{*}(\boldsymbol{b})$, where $\boldsymbol{b} \in H_{m}(H, \mathbb{Z})$ and $i: H \rightarrow G(p, m)$ denotes the inclusion. Then

$$
\boldsymbol{b}=\pi_{*}(\boldsymbol{a}(p, m))=\pi_{*} \circ\left(\phi_{p}\right)_{*}\left[\mathbb{T}^{m}\right]=\psi_{*} \circ \rho_{*}\left[\mathbb{T}^{m}\right]=\psi_{*}(0)=0
$$

as $\boldsymbol{b}=\pi_{*} \circ i_{*}(\boldsymbol{b})$. This gives a contradiction the class $\boldsymbol{a}(p, m)$ being nontrivial.

In order to conclude the proof, remark that

$$
\mathfrak{S}(\boldsymbol{a}(p, m))=\mathfrak{S}_{\phi_{p}}\left(\mathbb{T}^{m}\right) \leq \mathfrak{S}\left(\mathbb{T}^{m}\right) \leq 1,
$$

for any $m \geq 3$ and any prime $p$. 
This theorem implies the following unexpected result in dimensions $m \geq 4$.

Corollary 4.6 For any dimension $m \geq 4$, there exists an infinite number of irreducible orientable manifolds $M$ of dimension $m$ with pairwise nonisomorphic fundamental groups such that $\mathfrak{S}(M) \leq 1$.

Proof By construction, every class $\boldsymbol{a}(p, m)$ is representable by a manifold. If $m \geq 4$, such a manifold can be modified by surgery in order to get a new manifold denoted by $M(p, m)$ such that $\pi_{1}(M(p, m))=G(p, m)$ and $\Phi_{*}[M(p, m)]=\boldsymbol{a}(p, m)$, where $\Phi: M(p, m) \rightarrow K(G(p, m), 1)$ denotes the classifying map; see [3]. The infinite sequence of manifolds $\{M(p, m)\}$ where $p$ runs over all prime numbers gives an infinite sequence of irreducible orientable manifolds $M$ of dimension $m$ with pairwise nonisomorphic fundamental groups such that $\mathfrak{S}(M) \leq 1$.

The following natural question remains open:

Question 4.7 Consider the systolic volume $\mathfrak{S}(\cdot)$ as a function over all irreducible orientable manifolds of dimension $m$. Does there exist a positive number $C$ such that the number of distinct values of the function $\mathfrak{S}(\cdot)$ less than $C$ is infinite?

\section{Systolic volume of multiple classes}

Given a finitely presentable group $G$ and a homology class $\boldsymbol{a} \in H_{*}(G, \mathbb{Z})$, how does the function $\mathfrak{S}(G, k \boldsymbol{a})$ behave in terms of the integer variable $k$ ? In this section, we prove Theorem III which we restate here for the reader's convenience.

Theorem 5.1 Let $G$ be a finitely presentable group and $\boldsymbol{a} \in H_{m}(G, \mathbb{Z})$, where $m \geq 3$. Then there exists a positive number $C(G, \boldsymbol{a})$ depending only on the pair $(G, \boldsymbol{a})$ so that

$$
\mathfrak{S}(G, k \boldsymbol{a}) \leq C(G, \boldsymbol{a}) \cdot \frac{k}{\ln (1+k)}
$$

for any positive integer $k$.

Before proving this result, we put the question in a more general context.

\subsection{Systolic volume of the sum of homology classes}

Let $G_{1}$ and $G_{2}$ be two finitely presentable groups and denote by $i_{j}: G_{j} \hookrightarrow G_{1} * G_{2}$ for $j=1,2$ the natural monomorphisms. Fix two integer homology classes $\boldsymbol{a}_{1} \in$ $H_{m}\left(G_{1}, \mathbb{Z}\right)$ and $\boldsymbol{a}_{2} \in H_{m}\left(G_{2}, \mathbb{Z}\right)$. The natural isomorphism

$$
H_{m}\left(G_{1} * G_{2}, \mathbb{Z}\right) \simeq H_{m}\left(G_{1}, \mathbb{Z}\right) \oplus H_{m}\left(G_{2}, \mathbb{Z}\right)
$$

for positive $m$ allows us to denote by $\boldsymbol{a}_{1}+\boldsymbol{a}_{2}$ the class $\left(i_{1}\right)_{*}\left(\boldsymbol{a}_{1}\right)+\left(i_{2}\right)_{*}\left(\boldsymbol{a}_{2}\right)$. 
Proposition 5.2 If $m \geq 3$,

$$
\mathfrak{S}\left(G_{1} * G_{2}, a_{1}+a_{2}\right) \leq \mathfrak{S}\left(G_{1}, a_{1}\right)+\mathfrak{S}\left(G_{2}, a_{2}\right) .
$$

Proof For any $\varepsilon>0$ and for $j=1,2$ we choose a geometric cycle $\left(X_{j}, f_{j}\right)$ of dimension $m$ representing $\boldsymbol{a}_{j}$ and satisfying

$$
\mathfrak{S}_{f_{j}}\left(X_{j}\right) \leq \mathfrak{S}\left(G_{j}, \boldsymbol{a}_{j}\right)+\frac{1}{2} \varepsilon .
$$

The geometric cycle $\left(X_{1} \# X_{2}, f_{1} \# f_{2}\right)$ obtained as the connected sum of $\left(X_{1}, f_{1}\right)$ and $\left(X_{2}, f_{2}\right)$ represents the class $\boldsymbol{a}_{1}+\boldsymbol{a}_{2}$. By the comparison principle (see [3]),

$$
\mathfrak{S}_{f_{1} \# f_{2}}\left(X_{1} \# X_{2}\right) \leq \mathfrak{S}_{f_{1} \vee f_{2}}\left(X_{1} \vee X_{2}\right)=\mathfrak{S}_{f_{1}}\left(X_{1}\right)+\mathfrak{S}_{f_{2}}\left(X_{2}\right),
$$

where $\left(X_{1} \vee X_{2}, f_{1} \vee f_{2}\right)$ denotes the wedge sum of $\left(X_{1}, f_{1}\right)$ and $\left(X_{2}, f_{2}\right)$. As $\varepsilon$ can be chosen arbitrarily small, we get the result.

If $\boldsymbol{a}_{1}$ and $\boldsymbol{a}_{2}$ are two homology classes of dimension $m$ of the same group $G$, we deduce the following subadditivity property of the systolic volume.

Corollary 5.3 Let $\boldsymbol{a}_{1}$ and $\boldsymbol{a}_{2}$ be two classes of $H_{m}(G, \mathbb{Z})$ with $m \geq 3$. Then

$$
\mathfrak{S}\left(G, a_{1}+a_{2}\right) \leq \mathfrak{S}\left(G, a_{1}\right)+\mathfrak{S}\left(G, a_{2}\right) .
$$

Proof Indeed, if we denote by $\pi: G * G \rightarrow G$ the epimorphism defined by $\pi \circ i_{j}=\operatorname{id}_{G}$, then $\pi_{*}\left(\boldsymbol{a}_{1}+\boldsymbol{a}_{2}\right)=\boldsymbol{a}_{1}+\boldsymbol{a}_{2}$. By Proposition 3.4, we get the result.

As a direct consequence of this corollary, we derive that

$$
\mathfrak{S}(G, k \boldsymbol{a}) \leq k \cdot \mathfrak{S}(G, \boldsymbol{a})
$$

for any $\boldsymbol{a} \in H_{m}(G, \mathbb{Z})$ with $m \geq 3$ and any integer $k$. In particular the limit

$$
\lim _{k \rightarrow \infty} \frac{\mathfrak{S}(G, k \boldsymbol{a})}{k}
$$

exists. Theorem 5.1 permits us to conclude that this limit is always zero.

\subsection{Sublinear upper bound for the systolic volume of multiple classes}

Theorem 5.1 is related to the behavior of systolic volume for connected sum and is a direct consequence of the following result. 
Theorem 5.4 Let $X$ be a connected pseudomanifold of dimension $m \geq 3$ and denote by $\#_{k} X$ the connected sum of $k$ copies of $X$. There exists a positive number $C(X)$ depending only on the topology of $X$ such that

$$
\mathfrak{S}(\underset{k}{\#} X) \leq C(X) \cdot \frac{k}{\ln (1+k)}
$$

for any positive integer $k$. If $k$ is large enough, this last inequality (5-2) is satisfied for

$$
C(X)=m \cdot c(X) \cdot \ln c(X),
$$

where $c(X)$ stands for the minimal number of $m$-cubes in a cubical decomposition of $X$.

Recall that a cubical decomposition of $X$ is a family of $m$-dimensional embedded topological cubes covering $X$ and such that any nonempty intersection between two cubes occurs along a lower-dimensional face. In particular, any $(m-1)$-face belongs to exactly two $m$-cubes. The existence of such decompositions can be proved by considering a triangulation of $X$ and decomposing each $m$-simplex of this triangulation into $m+1 m$-cubes.

Remark 5.5 Theorem 5.4 substantially improves [5, Theorem A], and the upper bound (5-2) still holds for a sequence of cyclic covering, the details being similar to those considered in the sequel (compare with [5]).

Proof The proof relies on ideas and techniques appearing in [5]. We consider a minimal cubical decomposition $\Theta$ of $X$ with $c(X)$ elements. From each cube $\mathcal{C} \in \Theta$ we remove an open cube $\mathcal{C}^{\prime} \simeq 11 / 4,3 / 4\left[^{m} \subset[0,1]^{m} \simeq \mathcal{C}\right.$ and denote by $X^{\prime}$ the polyhedron thus obtained. Each set (called a sleeve of $X^{\prime}$ )

$$
\overline{\mathcal{C}}=\mathcal{C} \backslash \mathcal{C}^{\prime} \simeq \partial \mathcal{C} \times[0,1 / 2]
$$

is endowed with the product metric $g_{\partial \mathcal{C}} \times \frac{1}{N} d t$, where $g_{\partial \mathcal{C}}$ denotes the metric on $\partial \mathcal{C}$ induced by a realization of $\mathcal{C} \simeq[0,1]^{m} \subset \mathbb{R}^{m}$ in the standard Euclidean vector space and $N$ is a positive integer to be chosen later. Choose the metrics $\left\{g_{\partial \mathcal{C}}\right\}_{\mathcal{C} \in \Theta}$ to coincide on each nonempty intersection between two $m$-cubes, and denote by $g^{\prime}$ the Riemannian polyhedral metric thus obtained on $X^{\prime}$. By construction:

- The complex $X^{\prime}$ is homeomorphic to $X$ minus $c(X)$ disjoint open $m$-disks, and in particular $\pi_{1}\left(X^{\prime}\right)=\pi_{1}(X)$.

- $\operatorname{vol}\left(X^{\prime}, g^{\prime}\right)=m \cdot \frac{1}{N} \cdot c(X)$.

- The obvious retraction of $X^{\prime}$ onto $\Theta^{(m-1)}$ (where $\Theta^{(m-1)}$ denotes the $(m-1)$ skeleton of $\Theta$ ) is distance nonincreasing. 
Fix an $m$-cube $\mathcal{C} \in \Theta$. We define the $\operatorname{star} \overline{\operatorname{st}}(\mathcal{C})$ as the union of all cubes with nonempty intersection with $\mathcal{C}$ (including $\mathcal{C}$ itself). The open $\operatorname{star} \operatorname{st}(\mathcal{C})$ is then defined as the union of the interiors of all cubes having a nonempty intersection with $\mathcal{C}$, so the closure of $\operatorname{st}(\mathcal{C})$ is exactly $\overline{\operatorname{st}}(\mathcal{C})$. The following lemma will be important in what follows.

Lemma 5.6 If a relative curve $\gamma$ of $\left(X^{\prime}, \overline{\mathcal{C}}\right)$ is not entirely included in $X^{\prime} \cap \operatorname{st}(\mathcal{C})$, then $l_{g^{\prime}}(\gamma) \geq 2$.

Proof Let $p$ belongs to the boundary $\partial(\overline{\operatorname{st}}(\mathcal{C}))=\overline{\operatorname{st}}(\mathcal{C}) \backslash \operatorname{st}(\mathcal{C})$. Then

$$
d_{g^{\prime}}(p, \partial \mathcal{C}) \geq 1
$$

which proves the lemma.

Indeed, suppose that this is not the case. The point $p$ lies in the $(m-1)$-face $F$ of an $m$-cube $\mathcal{C}_{1} \subset \overline{\mathrm{st}}(\mathcal{C})$. But $F$ belongs to exactly two $m$-cubes $\mathcal{C}_{1}$ and $\mathcal{C}_{2}$. As $d_{g^{\prime}}(F, \partial \mathcal{C}) \leq d_{g^{\prime}}(p, \partial \mathcal{C})<1$, we conclude that $F \cap \mathcal{C} \neq \varnothing$ which implies that $\mathcal{C}_{2} \subset \overline{\operatorname{st}}(\mathcal{C})$. This together with the condition $d_{g^{\prime}}(p, \partial \mathcal{C})<1$ implies that $p \in \operatorname{st}(\mathcal{C})$ which is a contradiction.

According to the construction of graphs with large girth by Erdös and Sachs [15], there exists for any positive integers $n$ and $N$ such that

$$
n \geq 2 \sum_{t=1}^{N-2}(c(X)-1)^{t}
$$

a $c(X)$-regular graph $\Gamma$ with $2 n$ vertices whose girth - defined as the least number of edges composing a cycle - is at least $N$. The graph $\Gamma$ can be thought as a 1dimensional polyhedron on which we define the PL-metric $h$ for which all edges have length $1 / N$. The girth's bound thus says that $\operatorname{sys}(\Gamma, h) \geq 1$.

Denote by $v_{1}, \ldots, v_{2 n}$ the vertices of $\Gamma$ and let $\left(X_{1}^{\prime}, g_{1}^{\prime}\right), \ldots,\left(X_{2 n}^{\prime}, g_{2 n}^{\prime}\right)$ be $2 n$ copies of the Riemannian polyhedron $\left(X^{\prime}, g^{\prime}\right)$. We define a map $F: X_{1}^{\prime} \cup \cdots \cup X_{2 n}^{\prime} \rightarrow \Gamma$ as follows. For each $i=1, \ldots, 2 n$ :

- Set $F(p)=v_{i}$ if $p \in \Theta_{i}^{m-1}$, where $\Theta_{i}^{m-1}$ denotes the $(m-1)$-skeleton of the cubical decomposition $\Theta_{i}$ corresponding to $X_{i}^{\prime}$.

- Denote by $e_{1}^{i}, \ldots, e_{c(X)}^{i}$ the edges adjacent to $v_{i}$, and by $\overline{\mathcal{C}}_{1}^{i}, \ldots, \overline{\mathcal{C}}_{c(X)}^{i}$ the sleeves of $X_{i}^{\prime}$ corresponding to the cubical decomposition $\Theta_{i}$. For each $j=$ $1, \ldots, c(X)$, each edge $e_{j}^{i}$ is isometric to $\left([0,1], \frac{1}{N} d t\right)$ with 0 being identified with the vertex $v_{i}$. Then for each point $p=(x, t)$ belonging to the sleeve $\overline{\mathcal{C}}_{j}^{i} \simeq \partial \mathcal{C}_{j}^{i} \times\left[0, \frac{1}{2}\right]$ set $F_{i}(p)=t$.

Observe that each restriction $F_{\mid X_{i}^{\prime}}:\left(X_{i}^{\prime}, g_{i}^{\prime}\right) \rightarrow(\Gamma, h)$ is distance nonincreasing. 
Now to each edge $e=\left(v_{i}, v_{j}\right) \in E(\Gamma)$ correspond exactly two sleeves $\overline{\mathcal{C}}$ and $\overline{\mathcal{C}}^{\prime}$ belonging respectively to the two distinct copies $X_{i}^{\prime}$ and $X_{j}^{\prime}$ of $X^{\prime}$ and such that their images under $F$ cover the edge $e$. We choose a PL-isometry denoted by $\phi_{e}$ between $\left(\partial \mathcal{C} \times\left\{\frac{1}{2}\right\}, g_{i}^{\prime}\right)$ and $\left(\partial \mathcal{C}^{\prime} \times\left\{\frac{1}{2}\right\}, g_{j}^{\prime}\right)$. The quotient space

$$
X(n)=\left(X_{1}^{\prime} \cup \cdots \cup X_{2 n}^{\prime}\right) /\left\{\phi_{e}\right\}_{e \in E(\Gamma)}
$$

is a polyhedron homeomorphic to

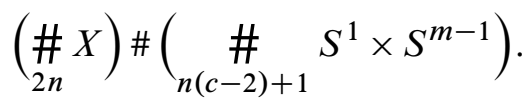

The metrics $\left\{g_{i}^{\prime}\right\}_{i=1, \ldots, n}$ are compatible with the quotient map $X_{1}^{\prime} \cup \cdots \cup X_{n}^{\prime} \rightarrow X(n)$ and give rise to a Riemannian polyhedral metric on $X(n)$ that we denote by $g(n)$. The map $F$ induces a new map still denoted by $F$ from $X(n)$ onto $\Gamma$. By construction this map $F:(X(n), g(n)) \rightarrow(\Gamma, h)$ is distance nonincreasing.

According to [5, Proposition 1] the addition of 1-handles does not change the value of the systolic volume, and therefore

$$
\mathfrak{S}(\underset{2 n}{\#} X)=\mathfrak{S}(X(n)) \leq \frac{\operatorname{vol}(X(n), g(n))}{\left(\operatorname{sys}(X(n), g(n))^{m}\right.} .
$$

We have

$$
\operatorname{vol}(X(n), g(n))=2 n \cdot \operatorname{vol}\left(X^{\prime}, g^{\prime}\right)=2 n \cdot m \cdot \frac{1}{N} \cdot c(X) .
$$

Lemma 5.7 We have

$$
\operatorname{sys}(X(n), g(n)) \geq 1 .
$$

Proof Let $\gamma:[0,1] \rightarrow X(n)$ be a closed curve which is not contractible.

If its image $F(\gamma)$ is not contractible in $\Gamma$, then

$$
l_{g(n)}(\gamma) \geq l_{h}(F(\gamma)) \geq \operatorname{sys}(\Gamma, h) \geq 1
$$

as $F$ is distance nonincreasing.

Now suppose that $F(\gamma)$ is contractible in $\Gamma$. We will show that $l_{g(n)}(\gamma) \geq 2$ using Lemma 5.6.

First of all, we can assume that $\gamma$ is minimizing in its own homotopy class. The contractibility of $F(\gamma)$ implies that we can find an edge $\left[v_{i}, v_{j}\right]$ of $\Gamma$ joining two vertices $v_{i}$ and $v_{j}$, a point $\left.\left.v \in\right] v_{i}, v_{j}\right]$ and a triplet $0 \leq t_{1}<t_{*}<t_{2} \leq 1$ such that:

- $v=F\left(\gamma\left(t_{*}\right)\right)$.

- $F\left(\gamma\left(\left[t_{1}, t_{2}\right]\right) \subset\left[v_{i}, v\right]\right.$.

- $F\left(\gamma\left(t_{1}\right)\right)=F\left(\gamma\left(t_{2}\right)\right)=v_{i}$. 
Denote by $m_{i j}$ the midpoint of the edge $\left[v_{i}, v_{j}\right]$, and by

$$
\overline{\mathcal{C}}_{i}=\overline{F^{-1}(] v_{i}, m_{i j}[)} \quad \text { and } \quad \overline{\mathcal{C}}_{j}=\overline{F^{-1}(] m_{i j}, v_{j}[)}
$$

the two corresponding sleeves.

If $\left.v \in] v_{i}, m_{i j}\right]$, we contract the portion of the curve $\{\gamma(t)\}_{t \in\left[t_{1}, t_{2}\right]}$ using the projection $\pi_{i}: \overline{\mathcal{C}}_{i} \simeq \partial \mathcal{C}_{i} \times\left[0, \frac{1}{2}\right] \rightarrow \partial \mathcal{C}_{i} \times\{0\}$ given by the formula $\pi_{i}(p, t)=(p, 0)$. This contraction strictly decreases the length of $\gamma$ which is in contradiction with its minimality. If $v \in] m_{i j}, v_{j}$ [, we contract the portion of the curve $\{\gamma(t)\}_{t \in\left[t_{1}, t_{2}\right]}$ lying in $\overline{\mathcal{C}}_{j}$ using the projection $\pi_{j}: \overline{\mathcal{C}}_{j} \simeq \partial \mathcal{C}_{j} \times\left[0, \frac{1}{2}\right] \rightarrow \partial \mathcal{C}_{j} \times\left\{\frac{1}{2}\right\}$ given by the formula $\pi_{j}(p, t)=\left(p, \frac{1}{2}\right)$. This contraction also strictly decreases the length of $\gamma$ which is again in contradiction with its minimality. So the point $v$ must coincide with $v_{j}$.

The restriction of $\gamma$ to the interval $\left[t_{1}, t_{2}\right]$ is thus the concatenation $\gamma_{1} \star \gamma_{2} \star \gamma_{3}$ of three curves with $\gamma_{1}, \gamma_{3} \subset F^{-1}\left(\left[v_{i}, v_{j}[)\right.\right.$ and $\gamma_{2} \subset F^{-1}\left(v_{j}\right)=\Theta_{j}^{m-1}$. We will show that $\gamma_{2}$ is not entirely contained in the open $\operatorname{star} \operatorname{st}\left(\mathcal{C}_{j}\right)$ corresponding to the sleeve $\overline{\mathcal{C}}_{j}$, and thus

$$
l_{g(n)}(\gamma) \geq l_{g_{j}^{\prime}}\left(\gamma_{2}\right) \geq 2
$$

according to Lemma 5.6 which concludes the proof of the lemma.

Indeed suppose that $\gamma_{2}$ is entirely contained in the open $\operatorname{star} \operatorname{st}\left(\mathcal{C}_{j}\right)$. Observe first that $\gamma_{2} \not \subset \overline{\mathcal{C}}_{j}$. Otherwise we contract the portion of the curve $\{\gamma(t)\}_{t \in\left[t_{1}, t_{2}\right]}$ lying in $\overline{\mathcal{C}}_{j}$ using the projection $\pi_{j}: \overline{\mathcal{C}}_{j} \simeq \partial \mathcal{C}_{j} \times\left[0, \frac{1}{2}\right] \rightarrow \partial \mathcal{C}_{j} \times\left\{\frac{1}{2}\right\}$ given by the formula $\pi_{j}(p, t)=\left(p, \frac{1}{2}\right)$. This contraction strictly decreases the length of $\gamma$ which is in contradiction with its minimality. Now the orthogonal projections of $\operatorname{st}\left(\overline{\mathcal{C}}_{j}\right) \cap \Theta_{j}^{m-1}$ on each face of $\overline{\mathcal{C}}_{j} \cap \Theta_{j}^{m-1}$ are correctly defined and coherent. So by projecting $\gamma_{2}$ orthogonally on $\overline{\mathcal{C}}_{j} \cap \Theta_{j}^{m-1}$ we do not change the homotopy class of $\gamma$, and since $\gamma_{2} \not \subset \overline{\mathcal{C}}_{j}$, the length of $\gamma$ strictly decreases. This is again a contradiction with its minimality.

So

$$
\mathfrak{S}(\underset{2 n}{\#} X) \leq \frac{\operatorname{vol}(X(n), g(n))}{\left(\operatorname{sys}(X(n), g(n))^{m}\right.} \leq m \cdot \frac{2 n}{N} \cdot c(X)
$$

for any positive integers $n$ and $N$ such that

$$
n \geq \frac{2}{c(X)-2}\left[(c(X)-1)^{N-1}-(c(X)-1)\right] .
$$

Fix a positive integer $n$. As $c(X) \geq 2 m+1 \geq 7$, we choose $N$ such that

$$
n \in\left[(c(X)-1)^{N-1},(c(X)-1)^{N}\right] \text {, }
$$


and get that

$$
\mathfrak{S}(\underset{2 n}{\#} X) \leq m \cdot c(X) \cdot \ln (c(X)-1) \cdot \frac{2 n}{\ln (2 n+1)}
$$

according to (5-4). Furthermore

$$
\begin{aligned}
\mathfrak{S}(\underset{2 n+1}{\#} X) & \leq \mathfrak{S}(\underset{2 n}{\# X)}+\mathfrak{S}(X) \\
& \leq m \cdot c(X) \cdot \ln (c(X)-1) \cdot \frac{2 n}{\ln (2 n+1)}+\mathfrak{S}(X) \\
& \leq m \cdot c(X) \cdot \ln (c(X)-1) \cdot \frac{2 n+1}{\ln (2 n+2)}+\mathfrak{S}(X) .
\end{aligned}
$$

For $n$ large enough, we thus get the universal upper bound (5-2) which concludes the proof.

\subsection{Homology classes with positive simplicial volume}

Recall the following definition (see Gromov [16]).

Definition 5.8 Let $X$ be a pseudomanifold of dimension $m$. Its simplicial volume is the quantity

$$
\|X\|_{\Delta}=\inf \left\{\sum_{i}\left|r_{i}\right| \mid[X]=\sum_{i} r_{i} \sigma_{i}^{m}\right\},
$$

where the infimum is taken over the set of representations of the fundamental class $[X]$ by singular simplicial chains with real coefficients.

If $G$ denotes a finitely presentable group and $\boldsymbol{a}$ a homology class of dimension $m$, the simplicial volume of $\boldsymbol{a}$ is then the number

$$
\|\boldsymbol{a}\|_{\Delta}=\inf \{\|X\| \mid X \text { representing } \boldsymbol{a}\} .
$$

For homology classes whose simplicial volume is positive, the function $\mathfrak{S}(G, k \boldsymbol{a})$ goes to infinity and the following result provides a better information about its asymptotic behavior.

Corollary 5.9 Let $G$ be a finitely presentable group and $\boldsymbol{a} \in H_{m}(G, \mathbb{Z})$ be a homology class of dimension $m \geq 3$ such that $\|\boldsymbol{a}\|_{\Delta}>0$. Then there exist two positive numbers $C(G, \boldsymbol{a})$ and $C^{\prime}(G, \boldsymbol{a})$ depending only on the pair $(G, \boldsymbol{a})$ such that

$$
C^{\prime}(G, \boldsymbol{a}) \cdot \frac{k}{(\ln (1+k))^{m}} \leq \mathfrak{S}(G, k \boldsymbol{a}) \leq C(G, \boldsymbol{a}) \cdot \frac{k}{\ln (1+k)}
$$

for any positive integer $k$. 
Proof The lower bound is a direct consequence of the following inequality of Gromov (see [17, Theorem 6.4. $\mathrm{D}^{\prime}$ ]): any pseudomanifold $X$ of dimension $m$ satisfies the inequality

$$
C_{m}^{\prime} \frac{\|X\|_{\Delta}}{\left(\ln \left(2+\|X\|_{\Delta}\right)\right)^{m}} \leq \mathfrak{S}(X),
$$

where $C_{m}^{\prime}$ is a positive number depending only on the dimension $m$. It remains to remark that, if $X$ represents the class $k a$, then $\|X\|_{\Delta}=\|k \boldsymbol{a}\|_{\Delta}=k\|\boldsymbol{a}\|_{\Delta}$. The upper bound then follows by Theorem 5.1.

\subsection{Large oscillations of systolic volume}

The following example shows that the function $k \mapsto \mathfrak{S}(G, k \boldsymbol{a})$ may have arbitrarily large oscillations.

Let $m=2 l+1 \geq 3$ be an odd integer and $q \geq 2$ an integer. Let $X$ be an essential manifold of dimension $m$ (for example aspherical) and $f: X \rightarrow K\left(\pi_{1}(X), 1\right)$ be its classifying map. If $X$ is not aspherical, we assume that the image of its fundamental class $f_{*}[X]$ is an element of infinite order in $H_{m}\left(\pi_{1}(X), \mathbb{Z}\right)$. Set $a=f_{*}[X]$. Fix a generator $\boldsymbol{b} \in H_{m}\left(\mathbb{Z}_{q}, \mathbb{Z}\right)=\mathbb{Z}_{q}$. For each $1 \leq l \leq q-1$, we choose a normal representation of $l \boldsymbol{b}$ by a manifold $Y_{l}$ with $\pi_{1}\left(Y_{l}\right)=\mathbb{Z}_{q}$. For relatively prime $l$ and $q$, the corresponding lens space can be chosen to be $Y_{l}$. Set

$$
D=\max _{1 \leq k \leq q} \mathfrak{S}\left(\pi_{1}(X), k \boldsymbol{a}\right)
$$

and fix any positive number $C$. Consider the free product

and pick in

$$
G_{n}=\pi_{1}(X) * \underbrace{\mathbb{Z}_{q} * \cdots * \mathbb{Z}_{q}}_{n},
$$

$$
H_{m}\left(G_{n}, \mathbb{Z}\right)=H_{m}\left(\pi_{1}(X), \mathbb{Z}\right) \oplus H_{m}\left(\mathbb{Z}_{q}, \mathbb{Z}\right) \oplus H_{m}\left(\mathbb{Z}_{q}, \mathbb{Z}\right) \oplus \cdots \oplus H_{m}\left(\mathbb{Z}_{q}, \mathbb{Z}\right)
$$

the class $\boldsymbol{c}=\boldsymbol{a} \oplus \underbrace{\boldsymbol{b} \oplus \ldots \oplus \boldsymbol{b}}_{n}$. If $X_{k}$ is a manifold representing the class $k \boldsymbol{a}$, then

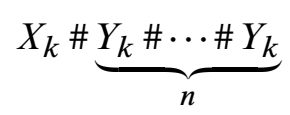

represents the class $k c$. By Corollary 3.5,

$$
\mathfrak{S}\left(G_{n}, k \boldsymbol{c}\right) \geq \mathfrak{S}(\underbrace{Y_{k} \# \cdots \# Y_{k}}_{n}) .
$$

Now [25, Theorem A] implies that if $n$ is chosen large enough, we have $\mathfrak{S}\left(G_{n}, k \boldsymbol{c}\right)>C$ for any $1 \leq k \leq q-1$. Besides $q \boldsymbol{c}=q \boldsymbol{a}$ in $H_{m}\left(G_{n}, \mathbb{Z}\right)$, and so $\mathfrak{S}\left(G_{n}, q \boldsymbol{c}\right) \leq D$. 


\subsection{Systolic generating function}

If $G$ is a finitely presentable group and $\boldsymbol{a} \in H_{m}(G, \mathbb{Z})$ is a homology class of infinite order, the study of the sequence $\{\mathfrak{S}(G, k \boldsymbol{a})\}_{k=1}^{\infty}$ is equivalent to the study of analytic properties of the following generating function:

$$
\sigma_{G, \boldsymbol{a}}(z)=\sum_{k=1}^{\infty} \mathfrak{S}(G, k \boldsymbol{a}) \cdot z^{k} .
$$

If the group $G$ is clearly identified by the context, we simplify the notation into $\sigma_{\boldsymbol{a}}(z)$. The upper bound (5-1) implies that $\sigma_{\boldsymbol{a}}(z)$ is an analytic function on the disk $|z|<1$. Furthermore the complex point $z=1$ is a singular point of this function as $\mathfrak{S}(G, k \boldsymbol{a}) \geq$ $\sigma_{m}>0$.

In general there is no hope for $\sigma_{G, \boldsymbol{a}}(z)$ to be a rational function. Indeed, if $\boldsymbol{a}$ is a class with positive simplicial volume, Corollary 5.9 teaches us that $z=1$ is not a pole of the corresponding systolic generating function, so $\sigma_{\boldsymbol{a}}(z)$ is not rational. It is well known that the rationality of the generating function of a numerical sequence is equivalent to the recurrence of this sequence. We deduce the following

Proposition 5.10 Let $G$ be a finitely presentable group and $\boldsymbol{a} \in H_{m}(G, \mathbb{Z})$ a homology class of infinite order. If the simplicial volume of $\boldsymbol{a}$ is positive, the sequence of systolic volumes $\{\mathfrak{S}(G, k \boldsymbol{a})\}_{k=1}^{\infty}$ does not satisfy any linear recurrence equation.

Nevertheless the rationality of $\sigma_{\boldsymbol{a}}(z)$ seems plausible for classes $\boldsymbol{a}$ with a bounded sequence of systolic volume $\{\mathfrak{S}(G, k \boldsymbol{a})\}_{k=1}^{\infty}$. Tori give a model of this type of behavior for multiple classes.

Proposition 5.11 Let $3 \leq m \leq n$ be two integers. Any class $\boldsymbol{a} \in H_{m}\left(\mathbb{Z}^{n}, \mathbb{Z}\right)$ satisfies the inequality

$$
\mathfrak{S}\left(\mathbb{Z}^{n}, \boldsymbol{a}\right) \leq\left(\begin{array}{l}
n \\
m
\end{array}\right) \cdot \mathfrak{S}\left(\mathbb{T}^{m}\right),
$$

where $\left(\begin{array}{l}n \\ m\end{array}\right)$ denotes the binomial coefficient.

Proof Fix a basis of $H_{m}\left(\mathbb{Z}^{n}, \mathbb{Z}\right)$ composed of embedded $m$-tori, and write the class $\boldsymbol{a}$ in this basis as

$$
\boldsymbol{a}=\sum_{i=1}^{\left(\begin{array}{c}
n \\
m
\end{array}\right)} k_{i}\left[\mathbb{T}_{i}^{m}\right],
$$


where $k_{i} \in \mathbb{Z}$ for each $i$. By Corollary 5.3,

$$
\mathfrak{S}(G, \boldsymbol{a}) \leq \sum_{i=1}^{\left(\begin{array}{c}
n \\
m
\end{array}\right)} \mathfrak{S}\left(G, k_{i}\left[\mathbb{T}_{i}^{m}\right]\right) .
$$

Observe that

$$
\mathfrak{S}\left(\mathbb{Z}^{n}, k\left[\mathbb{T}_{i}^{m}\right]\right) \leq \mathfrak{S}\left(\mathbb{T}^{m}\right)
$$

for any integer $k$. In fact, if $f: \mathbb{T}^{m} \rightarrow \mathbb{T}_{i}^{m}$ denotes a map of degree $k$, then the geometric cycle $\left(\mathbb{T}^{m}, f\right)$ represents the class $k\left[\mathbb{T}_{i}^{m}\right]$. By adding 1 -handles, we can normalize this representation into a geometric cycle $\left(\mathbb{T}^{m} \#\left(S^{1} \times S^{m-1}\right) \# \cdots \#\left(S^{1} \times\right.\right.$ $\left.\left.S^{m-1}\right), \tilde{f}\right)$. We get

$$
\begin{aligned}
\mathfrak{S}\left(\mathbb{Z}^{n}, k\left[\mathbb{T}_{i}^{m}\right]\right) & =\mathfrak{S}_{\tilde{f}}\left(\mathbb{T}^{m} \#\left(S^{1} \times S^{m-1}\right) \# \cdots \#\left(S^{1} \times S^{m-1}\right)\right) \\
& \leq \mathfrak{S}\left(\mathbb{T}^{m} \#\left(S^{1} \times S^{m-1}\right) \# \cdots \#\left(S^{1} \times S^{m-1}\right)\right)=\mathfrak{S}\left(\mathbb{T}^{m}\right) .
\end{aligned}
$$

Now we deduce the result by combining inequalities (5-5) and (5-6).

We close this chapter with the following.

Conjecture 5.12 If $\boldsymbol{a}=\left[\mathbb{T}^{m}\right] \in H_{m}\left(\mathbb{Z}^{m}, \mathbb{Z}\right)$, then the associated systolic generating function is

$$
\sigma_{\boldsymbol{a}}(z)=\mathfrak{S}\left(\mathbb{T}^{m}\right) \cdot \frac{z}{1-z} .
$$

\section{The Heisenberg group, nilmanifolds and the Waring problem}

The nilpotent groups give particularly interesting examples: the systolic volume of multiples of certain homology classes are bounded, albeit certain of these multiples admit (nonnormalized) representations by manifolds whose systolic volume is not bounded. This phenomena already appears in the simplest case of nilpotent nonabelian groups, that is the Heisenberg group.

\subsection{Nilmanifolds and the Waring problem}

Consider a nilpotent group $G$ of finite type without torsion. The classical result of Mal'cev [20] implies that there exists a simply connected nilpotent Lie group $\mathcal{G}(G)$ such that $G$ embeds in $\mathcal{G}(G)$ as a lattice, that is as a cocompact discrete subgroup. 
Denote by $\mathcal{L}(G)$ the Lie algebra of $\mathcal{G}(G)$ and suppose that $\mathcal{L}(G)$ is graded in the following way:

$$
\mathcal{L}(G)=\bigoplus_{k=1}^{s} \mathcal{L}_{k}, \quad\left[\mathcal{L}_{i}, \mathcal{L}_{j}\right] \subset \mathcal{L}_{i+j},
$$

where $\mathcal{L}_{i+j}=0$ if $i+j>s$. We do not suppose here that

$$
\left\{\mathcal{L}_{(i)}=\bigoplus_{k=i}^{s} \mathcal{L}_{k}\right\}_{i=1}^{s}
$$

is a lower central series, $s$ being not in general the nilpotency class of $\mathcal{L}(G)$. For any $t \in \mathbb{R}$, a natural homothety $\delta_{t}$ is associated to the decomposition (6-1) by the formula

$$
\delta_{t}(v)=t^{k} v \quad \text { if } v \in \mathcal{L}_{k} .
$$

This homothety $\delta_{t}$ is an endomorphism of $\mathcal{L}(G)$ for any real parameter $t$. By the Baker-Campbell-Hausdorff formula, and taking into account the structure (6-1), the homothety $\delta_{t}$ generates a homothety $\Delta_{t}$ of $\mathcal{G}$.

Definition 6.1 The nilpotent group $G$ is said to be graded if there exists a graduation (6-1) of the corresponding Lie algebra $\mathcal{L}(G)$ such that for integer parameters the corresponding homotheties of $\mathcal{G}(G)$ preserve the lattice $G \subset \mathcal{G}(G)$, that is

$$
\Delta_{n}(G) \subset G \text { for all } n \in \mathbb{Z} .
$$

If $G$ is a nilpotent graded group, let

$$
d(G)=\sum_{k=1}^{s} k \operatorname{dim} \mathcal{L}_{k}
$$

be the weighted dimension of its corresponding Lie algebra $\mathcal{L}(G)$. Remark that if the sequence of subalgebras (6-2) is the lower central series, $d(G)$ coincides with the degree of polynomial growth of $G$; see Wolf [28] and Bass [9].

Denote by $M=\mathcal{G}(G) / G$ the nilmanifold corresponding to the nilpotent group $G$. If $G$ is graded, the homothety $\Delta_{n}: \mathcal{G}(G) \rightarrow \mathcal{G}(G)$ defined using the graduation induces for every positive integer $n$ a map

$$
\widetilde{\Delta}_{n}: M \rightarrow M
$$

of degree $n^{d}$ with $d=d(G)$. Let $\boldsymbol{a}=[M] \in H_{m}(G, \mathbb{Z})$ be the fundamental class of $M$ and $k$ be a positive integer. We can represent the class $k \boldsymbol{a}$ by the connected 
sum of a uniformly bounded number of copies of $M$ as follows. By a result of Hilbert (see Ellison [13]), there exists an integer $\mathcal{K}(d)$ such that

$$
k=\sum_{i=1}^{s} a_{i}^{d}
$$

where each coefficient $a_{i}$ is a positive integer and $s \leq \mathcal{K}(d)$. Now the class $k \boldsymbol{a}$ is represented by the geometric cycle $\left(\#_{i=1}^{s} M_{i}, f\right)$, where $M_{i} \simeq M$ for $i=1, \ldots, s$ and

$$
f: \underset{i=1}{s} M_{i} \rightarrow \bigvee_{i=1}^{s} M_{i} \stackrel{\bigvee_{i=1}^{s} \widetilde{\Delta}_{a_{i}}}{\longrightarrow} M,
$$

the first map being the contraction of the connected sum into a wedge. We easily compute that $\operatorname{deg} f=\sum_{i=1}^{s} a_{i}^{d}=k$ and so

$$
\mathfrak{S}(G, k \boldsymbol{a}) \leq \mathfrak{S}\left(\#_{i=1}^{s} M\right) \text {. }
$$

Then we apply Proposition 3.6 in order to derive the following result.

Theorem 6.2 Let $G$ be a graded nilpotent group. If $\boldsymbol{a}=[\mathcal{G}(G) / G]$ denotes the fundamental class of the corresponding nilmanifold, then

$$
\mathfrak{S}(G, k \boldsymbol{a}) \leq \mathcal{K}(d(G)) \cdot \mathfrak{S}(G, \boldsymbol{a})
$$

for any positive integer $k$.

\subsection{Family of lattices in the Heisenberg group}

Consider the Heisenberg group of dimension 3 composed of the following set of upper triangular matrices:

$$
\mathcal{H}=\left\{\left(\begin{array}{lll}
1 & x & z \\
0 & 1 & y \\
0 & 0 & 1
\end{array}\right) \mid x, y, z \in \mathbb{R}\right\} .
$$

The subset $\mathcal{H}(\mathbb{Z})$ of matrices of $\mathcal{H}$ with integer coefficients (ie for which $x, y, z \in \mathbb{Z}$ ) is a lattice, and we denote by $M_{\mathcal{H}}=\mathcal{H} / \mathcal{H}(\mathbb{Z})$ the corresponding nilmanifold. The fundamental group $\mathcal{H}(\mathbb{Z})$ of $M_{\mathcal{H}}$ satisfies the assumptions of Theorem 6.2. In fact the homotheties $\left\{\Delta_{t}\right\}_{t>0}$ are given by the formula

$$
\Delta_{t}\left(\begin{array}{ccc}
1 & x & z \\
0 & 1 & y \\
0 & 0 & 1
\end{array}\right)=\left(\begin{array}{ccc}
1 & t x & t^{2} z \\
0 & 1 & t y \\
0 & 0 & 1
\end{array}\right),
$$


so $\Delta_{n}(\mathcal{H}(\mathbb{Z})) \subset \mathcal{H}(\mathbb{Z})$ for any integer $n \geq 1$. The map $\Delta_{n}$ factorizes through a map

$$
\tilde{\Delta}_{n}: M_{\mathcal{H}} \rightarrow M_{\mathcal{H}}
$$

for which $\operatorname{deg}\left(\widetilde{\Delta}_{n}\right)=n^{4}$. The resolution of the Waring problem for the sum of fourth powers $($ see $[7 ; 8])$ gives that any integer number decomposes into a sum of at most 19 fourth powers. That is, with the notation of Theorem 6.2, we have $d(\mathcal{H}(\mathbb{Z}))=4$ and $\mathcal{K}(4)=19$. Theorem IV of the introduction now easily follows.

Corollary 6.3 Let $\boldsymbol{a}=\left[M_{\mathcal{H}}\right] \in H_{3}(\mathcal{H}(\mathbb{Z}), \mathbb{Z})$ be the fundamental class of $M_{\mathcal{H}}$. Then

$$
\mathfrak{S}(\mathcal{H}(\mathbb{Z}), k \boldsymbol{a}) \leq 19 \cdot \mathfrak{S}(\mathcal{H}(\mathbb{Z}), \boldsymbol{a})
$$

for any positive integer $k$.

The different lattices of $\mathcal{H}$ give rise to nilmanifolds whose systolic behavior is particularly interesting. Consider the sequence of lattices $\left\{\mathcal{H}_{n}(\mathbb{Z})\right\}_{n=1}^{\infty}$ of $\mathcal{H}$, where $\mathcal{H}_{n}(\mathbb{Z})$ is the subset of matrices of $\mathcal{H}$ such that $x \in n \mathbb{Z}$ and $y, z \in \mathbb{Z}$. Denote by $M_{n}=M_{\mathcal{H}_{n}}=\mathcal{H} / \mathcal{H}_{n}(\mathbb{Z})$ the corresponding nilmanifolds. The manifold $M_{n}$ is a cyclic covering of $M_{\mathcal{H}}$ with $n$ sheets, so

$$
\mathfrak{S}\left(M_{n}\right) \leq C \frac{n}{\ln n},
$$

according to the version of Theorem 5.4 for cyclic coverings. The fact that the function $\mathfrak{S}\left(M_{n}\right)$ goes to infinity is not obvious. For instance the simplicial volume of these manifolds is zero, and thus the corresponding lower bound (see Corollary 5.9) does not apply. We are nevertheless able to prove the following proposition; see [6].

Proposition 6.4 [6] The function $\mathfrak{S}\left(M_{\mathcal{H}_{n}}\right)$ satisfies the inequality

$$
\mathfrak{S}\left(M_{\mathcal{H}_{n}}\right) \geq a \frac{\ln n}{\exp (b \sqrt{\ln \ln n})},
$$

where $a$ an $b$ are two positive constants. In particular,

$$
\lim _{n \rightarrow+\infty} \mathfrak{S}\left(M_{\mathcal{H}_{n}}\right)=+\infty
$$

As the cover $M_{n} \rightarrow M_{\mathcal{H}}$ has $n$ sheets, the manifold $M_{n}$ represents the class $n\left[M_{\mathcal{H}}\right] \in$ $H_{3}(\mathcal{H}(\mathbb{Z}), \mathbb{Z})$ for any positive integer $n$. This representation is not normalized, and Corollary 6.3 together with Proposition 6.4 shows that the assumption of normalization cannot be dropped in Proposition 2.4. 


\section{References}

[1] N A Baas, On bordism theory of manifolds with singularities, Math. Scand. 33 (1973) 279-302 MR0346824

[2] I K Babenko, Asymptotic invariants of smooth manifolds, Izv. Ross. Akad. Nauk Ser. Mat. 56 (1992) 707-751 MR1208148 In Russian; translated in Russian Acad. Sci. Izv. Math. 41 (1993) 1-38

[3] I K Babenko, Topologie des systoles unidimensionnelles, Enseign. Math. 52 (2006) 109-142 MR2255530

[4] I Babenko, Addenda à l'article intitulé "Topologie des systoles unidimensionnelles", Enseign. Math. 54 (2008) 397-398 MR2478093

[5] I Babenko, F Balacheff, Géométrie systolique des sommes connexes et des revêtements cycliques, Math. Ann. 333 (2005) 157-180 MR2169832

[6] I Babenko, F Balacheff, G Bulteau, Systolic geometry and simplicial complexity of groups arXiv:1501.01173

[7] R Balasubramanian, J-M Deshouillers, F Dress, Problème de Waring pour les bicarrés, I: Schéma de la solution, C. R. Acad. Sci. Paris Sér. I Math. 303 (1986) 85-88 MR853592

[8] R Balasubramanian, J-M Deshouillers, F Dress, Problème de Waring pour les bicarrés, II: Résultats auxiliaires pour le théorème asymptotique, C. R. Acad. Sci. Paris Sér. I Math. 303 (1986) 161-163 MR854724

[9] H Bass, The degree of polynomial growth of finitely generated nilpotent groups, Proc. London Math. Soc. 25 (1972) 603-614 MR0379672

[10] M Brunnbauer, Homological invariance for asymptotic invariants and systolic inequalities, Geom. Funct. Anal. 18 (2008) 1087-1117 MR2465685

[11] P Buser, P Sarnak, On the period matrix of a Riemann surface of large genus, Invent. Math. 117 (1994) 27-56 MR1269424

[12] J Cerf, Topologie de certains espaces de plongements, Bull. Soc. Math. France 89 (1961) 227-380 MR0140120

[13] W J Ellison, Waring's problem, Amer. Math. Monthly 78 (1971) 10-36 MR0414510

[14] D B A Epstein, The degree of a map, Proc. London Math. Soc. 16 (1966) 369-383 MR0192475

[15] P Erdös, H Sachs, Reguläre Graphen gegebener Taillenweite mit minimaler Knotenzahl, Wiss. Z. Martin-Luther-Univ. Halle-Wittenberg Math.-Natur. Reihe 12 (1963) 251-257 MR0165515

[16] M Gromov, Volume and bounded cohomology, Inst. Hautes Études Sci. Publ. Math. (1982) 5-99 MR686042 
[17] M Gromov, Filling Riemannian manifolds, J. Differential Geom. 18 (1983) 1-147 MR697984

[18] M Gromov, Systoles and intersystolic inequalities, from: "Actes de la Table Ronde de Géométrie Différentielle", (A L Besse, editor), Sémin. Congr. 1, Soc. Math. France, Paris (1996) 291-362 MR1427763

[19] F Hirzebruch, Komplexe Mannigfaltigkeiten, from: "Proc. Internat. Congress Math. 1958", Cambridge Univ. Press (1960) 119-136 MR0123733

[20] A I Mal' cev, On a class of homogeneous spaces, Izvestiya Akad. Nauk. SSSR. Ser. Mat. 13 (1949) 9-32 MR0028842

[21] J Milnor, On the cobordism ring $\Omega^{*}$ and a complex analogue, I, Amer. J. Math. 82 (1960) 505-521 MR0119209

[22] S P Novikov, Homotopy properties of Thom complexes, Mat. Sb. 57 (99) (1962) 407442 MR0157381

[23] Y B Rudyak, S Sabourau, Systolic invariants of groups and 2-complexes via Grushko decomposition, Ann. Inst. Fourier (Grenoble) 58 (2008) 777-800 MR2427510

[24] S Sabourau, Systolic volume and minimal entropy of aspherical manifolds, J. Differential Geom. 74 (2006) 155-176 MR2260931

[25] S Sabourau, Systolic volume of hyperbolic manifolds and connected sums of manifolds, Geom. Dedicata 127 (2007) 7-18 MR2338511

[26] R E Stong, Notes on cobordism theory, Princeton Univ. Press (1968) MR0248858

[27] R Thom, Quelques propriétés globales des variétés différentiables, Comment. Math. Helv. 28 (1954) 17-86 MR0061823

[28] J A Wolf, Growth of finitely generated solvable groups and curvature of Riemanniann manifolds, J. Differential Geometry 2 (1968) 421-446 MR0248688

Institut de Mathématiques et de Modélisation de Montpellier, Université Montpellier 2 Case Courrier 051, Place Eugène Bataillon, 34095 Montpellier Cedex, France

Laboratoire Paul Painlevé, Université Lille 1

Bâtiment M2, Cité Scientifique, 59655 Villeneuve-d'Ascq Cedex, France

babenko@math.univ-montp2.fr, florent.balacheff@math.univ-lille1.fr

Received: 12 November 2013 Revised: 10 July 2014 
